\title{
PLANE CURVES OF MINIMAL DEGREE WITH PRESCRIBED SINGULARITIES
}

\author{
GERT-MARTIN GREUEL, CHRISTOPH LOSSEN, AND EUGENII SHUSTIN
}

\begin{abstract}
We prove that there exists a positive $\alpha$ such that for any integer $d \geq 3$ and any topological types $S_{1}, \ldots, S_{n}$ of plane curve singularities, satisfying $\mu\left(S_{1}\right)+\cdots+\mu\left(S_{n}\right) \leq \alpha d^{2}$, there exists a reduced irreducible plane curve of degree $d$ with exactly $n$ singular points of types $S_{1}, \ldots, S_{n}$, respectively. This estimate is optimal with respect to the exponent of $d$. In particular, we prove that for any topological type $S$ there exists an irreducible polynomial of degree $d \leq 14 \sqrt{\mu(S)}$ having a singular point of type $S$.
\end{abstract}

\section{INTRODUCTION}

Throughout the article we consider all objects to be defined over an algebraically closed field $\mathbf{K}$ of characteristic zero.

In the paper we deal with the following classical problem: given an integer $d \geq 3$ and types $S_{1}, \ldots, S_{n}$ of plane curve singularities, does there exist a reduced irreducible plane curve of degree $d$ with exactly $n$ singular points of types $S_{1}, \ldots, S_{n}$, respectively? The complete answer is known for nodal curves [Sev]: an irreducible curve of degree $d$, with $n$ nodes as its only singularities, exists if and only if

$$
0 \leq n \leq \frac{(d-1)(d-2)}{2} .
$$

For other singularities, even for ordinary cusps there is no complete answer. Namely, various restrictions are found (from Plücker formulae to inequalities by Varchenko Var and Ivinskis [HiF, Ivi]), which read

$$
\sum_{i=1}^{n} \sigma\left(S_{i}\right)<\alpha_{2} d^{2}+\alpha_{1} d+\alpha_{0}, \quad \alpha_{2}=\text { const }>0,
$$

with some positive invariants $\sigma$ of singular points which are at most quadratic in d. We want to give an asymptotically optimal sufficient existence condition, that is a condition of type

$$
\sum_{i=1}^{n} \sigma\left(S_{i}\right)<\alpha d^{2}+o\left(d^{2}\right), \quad \alpha \leq \alpha_{2}
$$

providing (0.1) is necessary.

Note that an asymptotically exact condition, that is $\alpha=\alpha_{2}$, is hardly attainable. For example, there exist curves of degree $d=2 \cdot 3^{k},(k=1,2, \ldots)$ with $9\left(9^{k}-1\right) / 8=$ $9 d^{2} / 32+O(d)$ ordinary cusps Hi1]. But here the number of conditions imposed 
by the cusps is $d^{2} / 16+O(d)$ more than the dimension of the space of curves of degree $d$, therefore one cannot expect that all intermediate quantities of cusps may be realized.

The only previously known general sufficient condition for the existence of a curve with given singularities was (see [Shu]

$$
\sum_{i=1}^{n}\left(\mu\left(S_{i}\right)+4\right)\left(\mu\left(S_{i}\right)+5\right) \leq \frac{(d+3)^{2}}{2} .
$$

It is not asymptotically optimal, because the left-hand side may be about $d^{4}$.

The goal of this paper is:

Theorem 1. For any integer $d \geq 1$ and topological types $S_{1}, \ldots, S_{n}$ of plane curve singularities, satisfying

$$
\sum_{i=1}^{n} \mu\left(S_{i}\right) \leq \frac{d^{2}}{392}
$$

there exists a reduced irreducible plane projective curve of degree d with exactly $n$ singular points of types $S_{1}, \ldots, S_{n}$, respectively.

This estimate is asymptotically optimal, because always

$$
\sum_{i=1}^{n} \mu\left(S_{i}\right)<d^{2}
$$

The constant in (0.3) is not the best possible. Our method could give a bigger constant, providing more tedious computations. For certain classes of singularities such as simple or ordinary, there are much better results (see, for instance, GLS, section 3.3 and $[$ Shu $)$.

The problem is of interest even for one individual singularity. Given a singularity $S$, what is the minimal degree of a reduced irreducible plane projective curve having this singularity at the origin? The classical upper bound is the determinacy bound $\mu(S)+2$ [Tou, whereas a lower bound is $\sqrt{\mu(S)}+1$ (coming from intersecting two generic polars and Bézout's Theorem). We claim

Theorem 2. For any topological type $S$ of plane curve singularities there exists a reduced irreducible plane projective curve of degree $\leq 14 \sqrt{\mu(S)}$ with singularity $S$ at the origin.

We should like to thank the Deutsche Forschungsgemeinschaft and the grant G 039-304.01/95 of the German-Israeli Foundation for financial support.

\section{Strategy of the Proof}

To emphasize what is new in our approach we describe shortly the main previously known constructions. 
The first one is to construct, somehow, a curve of the given degree, which is degenerate with respect to the required curve, and then to deform it in order to obtain the prescribed singularities. For example, Severi [Sev] showed that singular points of a nodal curve, irreducible or not, can be smoothed out or preserved independently. Hence, taking the union of generic straight lines and smoothing out suitable intersection points, one obtains irreducible curves with any prescribed number of nodes, allowed by Plücker's formulae. Attempts to extend this construction on other singularities give curves with a number of singularities bounded from above by a linear function of the degree $d$ (see, for example, GrM for curves with nodes, cusps and ordinary triple points), because of the very restrictive requirement of the independence of deformations of singular points.

The second way consists of a construction especially adapted to the given degree and given collection of singularities. It may be based on a sequence of rational transformations of the plane applied to a more or less simple initial curve in order to get the required curve. Or it may consist in an invention of a polynomial defining the required curve. This can be illustrated by constructions of singular curves of small degrees as, for instance, in [W11, [W12], or by the construction of cuspidal curves as in Hi1, cited in the Introduction. Two main difficulties do not allow the appliance of this approach to a wide class of degrees and singularities: (1) for any new degree or singularity one has to invent a new construction, (2) even if one has constructed a curve with a lot of singularities, like in [Hi1], it is hard to check that these singular points can be smoothed out independently and any intermediate numbers of singularities can be realized.

Another idea, based on a modification of the Viro method of gluing polynomials (see the original method in $[\mathrm{Vir}]$ ) and on the independence of singular point deformations, was suggested in [Shu. This method, from the very beginning, requires a collection of "base curves" with given singularities (as, for instance, in Theorem 2), which originally provides only non-optimal results such as (0.2) for arbitrary singularities.

In our proof we use the previous constructions and introduce the following new element. With reduced germs of plane curves we associate a class $\mathcal{G S}$ of irreducible zero-dimensional schemes, called below generalized singularity schemes. Further we proceed in three main steps.

Step 1. Given a topological type $S$ of plane curve singularities, we show that there exists a scheme $X \in \mathcal{G S}$ with $\operatorname{deg} X \leq a_{1} \mu(S), a_{1}=$ const $>0$ such that the relation

$$
h^{1}\left(\mathbf{P}^{2}, \mathcal{J}_{X / \mathbf{P}^{2}}(d)\right)=0,
$$

where $\mathcal{J}_{X / \mathbf{P}^{2}} \subset \mathcal{O}_{\mathbf{P}^{2}}$ is the ideal sheaf of $X$, suffices for the existence of a curve of degree $d$ with a singular point of type $S$ (see Lemmas 5.1, 5.8 below).

Step 2. For our purposes we have to provide the previous $h^{1}$-vanishing as $d \leq a_{2} \sqrt{\operatorname{deg} X}, a_{2}=$ const $>0$. To do this, we observe that in the first step, $X$ can be replaced by a generic scheme $X^{\prime}$ in the same Hilbert scheme. Then we 
follow basically Hirschowitz [HiA], who obtained, in an analogous manner, the $h^{1}$ vanishing for schemes of generic fat points in the plane. Namely, we fix a straight line $L$ and apply an inductive procedure described in Sections 3 and 4, which consists of a passage from $X$ and $d$ to the residue scheme $X: L$ (called below the reduction of $X$ ) and $d-1$. Each time we have to verify that $X: L$ belongs to $\mathcal{G S}$ (Proposition 2.11), and that

$$
a_{3} d \leq \operatorname{deg}(X \cap L) \leq d+1, \quad a_{3}=\text { const }>0 .
$$

The latter relation is achieved by means of two operations: specialization of the scheme $X$ with respect to $L$ (Lemma 2.14), and extension of $X$ (Definitions 2.21. 2.23, Lemma 2.22) when the specialization fails.

Step 3. The final stage is a construction of curves with many singular points, done by means of a version of the Viro method (Section 6). Given topological singularities $S_{1}, \ldots, S_{n}$, we find curves of degrees

$$
d_{i} \leq a_{4} \sqrt{\mu\left(S_{i}\right)}, \quad i=1, \ldots, n, \quad a_{4}=\text { const }>0,
$$

each having a singular point of the corresponding type. Then we take a curve of degree

$$
d \leq a_{5} \sqrt{d_{1}^{2}+\ldots+d_{n}^{2}}, \quad a_{5}=\text { const }>0,
$$

with $n$ generic points of multiplicities $d_{1}, \ldots, d_{n}$, respectively, and deform these points in order to obtain the given singularities on a curve of any degree

$$
d \geq a_{6} \sqrt{\mu\left(S_{1}\right)+\ldots+\mu\left(S_{n}\right)}, \quad a_{6}=\text { const }>0 .
$$

\section{Singularity Schemes, Reductions and Extensions}

Throughout this section, $S$ denotes a smooth surface, $z \in S$, and $C$ a reduced curve on $S$. Since our statements are local, we may assume that $C$, or the germ $(C, z)$, is given by a power series which, by abuse of notation, is also denoted by $C$ or by $(C, z)$. If $z \notin C$, then $(C, z)$ denotes the empty germ or a unit of $\mathcal{O}_{S, z}$. Later, $z$ denotes also a finite set of points of $S$ and $(C, z)$ the corresponding multigerm.

Definition 2.1. The multiplicity of $C$ at $z$ is the non-negative integer

$$
\operatorname{mt}(C, z)=\max \left\{n \in \mathbf{Z} \mid C \in \mathfrak{m}_{z}^{n}\right\},
$$

where $\mathfrak{m}_{z}$ is the maximal ideal of $\mathcal{O}_{S, z}$, the analytic local ring of $S$ at $z$.

If $z \in C$, we define, as usual, (cf. [Zar], |Wah], [Tei], [BrK]) the topological type (or equisingularity type) of the germ $(C, z)$ by the following discrete characteristic: the embedded resolution tree of $(C, z)$ and the multiplicities of the total transforms of $(C, z)$ at infinitely near points (including $z$ ). Two germs with the same topological types are called equivalent (notation $\sim$ ).

Definition 2.2. $z$ is called an essential point of $C$ if $z \in C$, and if the germ $(C, z)$ is not smooth. If $z \in C$ and if $q \neq z$ is infinitely near to $z$, we denote by $C_{(q)}$, respectively $\widehat{C}_{(q)}$, the corresponding strict, respectively total, transforms under the 
composition of blowing-ups $\pi_{(q)}: S_{(q)} \longrightarrow S$ defining $q$. We call $q$ essential if it is not a node (ordinary double point) of the union of $C_{(q)}$ with the reduced exceptional divisor.

We shall introduce now the singularity scheme, respectively the generalized singularity scheme, of $(C, z)$, which are zero-dimensional subschemes of $S$ and which encode to a certain extent the topological type of $(C, z)$, respectively together with some higher order tangencies.

For $z \in C$ let $T(C, z)$ denote the (infinite) complete embedded resolution tree of $(C, z)$ with vertices the points infinitely near to $z$. It is naturally oriented, inducing a partial ordering on its vertices such that $z<q$ for all $q \in T(C, z) \backslash\{z\}$. If $z \notin C$ we define $T(C, z)$ to be the empty tree. Moreover, let

$$
T^{*}(C, z):=\{q \in T(C, z) \mid q \text { is essential }\}
$$

denote the tree of essential points of $(C, z)$, which is a finite subtree of $T(C, z)$.

Definition 2.3. Let $T^{*} \subset T(C, z)$ be a finite, connected subtree containing the essential tree $T^{*}(C, z)$. For any point $q \in T^{*}$ and any $f \in \mathcal{O}_{S, z}$ denote by $f_{(q)}$, respectively $\hat{f}_{(q)}$, the strict, respectively total, transform under the modification $\pi_{(q)}$ defining $q$. Put $m_{q}:=\operatorname{mt}\left(C_{(q)}, q\right), \hat{m}_{q}:=\operatorname{mt}\left(\widehat{C}_{(q)}, q\right)$ and define the ideal

$$
J:=J\left(C, T^{*}\right):=\left\{f \in \mathcal{O}_{S, z} \mid \operatorname{mt}\left(\hat{f}_{(q)}, q\right) \geq \hat{m}_{q}, \quad q \in T^{*}\right\} \subset \mathcal{O}_{S, z}
$$

and the subscheme of $S$ defined by $J$,

$$
X:=X\left(C, T^{*}\right)=Z(J), \quad \mathcal{O}_{X, z}:=\mathcal{O}_{S, z} / J
$$

which is concentrated on $\{z\} . X$ is called a generalized singularity (scheme) and the class of zero-dimensional subschemes of $S$, constructed in this way, is denoted by $\mathcal{G S}$. The subclass of schemes $X \in \mathcal{G S}$ with $T^{*}=T^{*}(C, z)$ is denoted by $\mathcal{S}, X \in \mathcal{S}$ is called a singularity (scheme).

Examples. 1. Let $(C, z)$ be smooth. If $T^{*}=\emptyset$, we obtain $J=\mathcal{O}_{S, z}$ and $X=\emptyset$. If $T^{*}=\left\{z=q_{0}, q_{1}, \ldots, q_{n}\right\}$ and $C=y$ with respect to local coordinates $(x, y)$ at $z$, then $J=\left\langle y, x^{n+1}\right\rangle$.

2. If $(C, z)$ is an ordinary $r$-fold singularity ( $r$ smooth branches with different tangents) and if $T^{*}=\{z\}\left(=T^{*}(C, z)\right)$ then $J=\mathfrak{m}_{z}^{r}$.

The following lemma shows the relation of $X$ to equisingular deformations of $(C, z)$. Note that the germ $(C, z)$ defining $X$ is not uniquely determined by $X$, and that the tree $T^{*}$ is part of the data of $X$. We write $J_{X}$ and $T_{X}^{*}$ for the ideal $J$ and the tree $T^{*}$ belonging to $X$.

By the following lemma, though $(C, z)$ is not uniquely determined by $X$, all topological invariants of $(C, z)$ can be associated uniquely to $X$. In particular, we define the multiplicity $\mathrm{mt} X$, the Milnor number $\mu(X)$ and the $\delta$-invariant $\delta(X)$ as those of $(C, z)$. 
Lemma 2.4. Let $X \in \mathcal{G S}$ be a generalized singularity scheme.

(i) If $X$ is defined by $(C, z)$ then a generic element of $J_{X}$ is topologically equivalent to $(C, z)$.

(ii) The set of base points of the ideal $J_{X}$ is equal to $T_{X}^{*}$, that is, the strict transforms of two generic elements in $J_{X}$ intersect exactly in $T_{X}^{*}$.

Proof. Adding a generic element $f \in J_{X}$ to the equation of $(C, z) \subset(S, z)$ defines another generic element of $J_{X}$ having exactly the given multiplicities $\hat{m}_{q}, q \in T^{*}$, in particular, it is topologically equivalent to $(C, z)$, since $T^{*}(C, z) \subset T^{*}$.

Moreover, this shows that the strict transforms of two generic elements have the same multiplicity $m_{q} \geq 1$ at $q \in T_{X}^{*}$. On the other hand, let $q \in T(C, z) \backslash T_{X}^{*}, Q$ be the corresponding branch of $(C, z)$ and $\bar{q}$ the predecessor of $q$ in $T(C, z)$. Then, slightly changing the tangent direction of the strict transform of $Q$ in $\bar{q}$, blowing down and composing with the other branches of $(C, z)$ defines an element of $J_{X}$ whose strict transform at $q$ does not contain $q$.

The concepts developed so far generalize immediately to multigerms $(C, z)$, $z=\left\{z_{1}, \ldots, z_{k}\right\} \subset S$. Then $T(C, z)$ and $T^{*}(C, z)$ are finite unions of trees. For $T^{*}(C, z) \subset T^{*} \subset T(C, z)$ such that $T^{*} \cap T\left(C, z_{i}\right)$ is a finite and connected subtree, we can define $J\left(C, T^{*}\right) \subset \mathcal{O}_{S, z}=\prod_{i=1}^{k} \mathcal{O}_{S, z_{i}}$ and $X=X\left(C, T^{*}\right)=Z(J)$, as before. $X$ is then a reducible subscheme of $S$, concentrated on $z_{1}, \ldots, z_{k}$. Let mt $X, \mu(X), \delta(X)$ denote the sum of the corresponding invariants at $z_{1}, \ldots, z_{k}$. We need this generalization after blowing up.

Let $z \in C \subset S$ be a point and $\widehat{S} \longrightarrow S$ be the blowing-up of $z$. We denote by $\widehat{C}$, respectively $C^{*}$, the total, respectively strict, transform of $C, E$ the reduced exceptional divisor and $\hat{z}:=E \cap C^{*}$. $\left(C^{*}, \hat{z}\right) \subset(\widehat{S}, \hat{z})$ is a (multi)germ.

For any $f \in \mathcal{O}_{S, z}$ satisfying $\operatorname{mt}(f, z) \geq m:=\operatorname{mt}(C, z)$ we may divide the total transform $\hat{f}$ by the $m$ 'th power of $E$ and we shall denote this multigerm at $\hat{z}$ by $\hat{f}: m E$. If $m=\operatorname{mt}(f, z)$, then $\hat{f}: m E=f^{*}$ is the strict transform of $f$. Note that for $q \in T(C, z) \backslash\{z\}$

$$
\operatorname{mt}\left(\hat{f}_{(q)}, q\right)=\operatorname{mt}\left((\hat{f}: m E)_{(q)}, q\right)+k(q) \cdot m,
$$

where $k(q) \in \mathbf{N}$ is independent of $f$. This holds especially for $f=C$, hence we obtain for $T^{*}(C, z) \subset T^{*} \subset T(C, z)$ :

Lemma 2.5. With the above notations:

$$
f \in J\left(C, T^{*}\right) \Leftrightarrow\left(\operatorname{mt}(f, z) \geq m \text { and } \hat{f}: m E \in J\left(C^{*}, T^{*} \backslash\{z\}\right)\right) .
$$

Let us denote by $\operatorname{mt}(X, q):=m_{q}, q \in T_{X}^{*}$, the multiplicity of $\mathbf{X}$ at $\mathbf{q}$ and by $\operatorname{deg}(X):=\operatorname{dim}_{K}\left(\mathcal{O}_{S, z} / J_{X}\right)$ the degree of $\mathbf{X}$.

Lemma 2.6. For $X \in \mathcal{G S}, T^{*}=T_{X}^{*}$ and $m_{q}=\operatorname{mt}(X, q)$ we have

$$
\operatorname{deg}(X)=\sum_{q \in T^{*}} \frac{m_{q}\left(m_{q}+1\right)}{2}=\delta(X)+\sum_{q \in T^{*}} m_{q} .
$$


Proof. The second equality follows from

$$
\delta(C, z)=\sum_{q \in T^{*}} \frac{m_{q}\left(m_{q}-1\right)}{2} .
$$

For the proof of the first one, cf. Cas, Proposition 6.1.

Lemma 2.7. Let $X \in \mathcal{S}$ be defined by a germ $(C, z)$ and let $I^{\text {es }} \subset \mathcal{O}_{S, z}$ be the equisingularity ideal of $(C, z)$ in the sense of Wahl ([Wah], cf. also [DiH]), then $I^{e s} \supset J_{X}$.

Proof. Clear from the definitions.

Lemma 2.8. Let $Q_{1}, \ldots, Q_{r}$ denote the branches of $(C, z)$ and $J=J\left(C, T^{*}\right)$. Then

$$
f \in J \Leftrightarrow\left(f, Q_{j}\right) \geq 2 \delta\left(Q_{j}\right)+\sum_{i \neq j}\left(Q_{i}, Q_{j}\right)+\sum_{q \in T^{*} \cap Q_{j}} \operatorname{mt}\left(Q_{j,(q)}, q\right),
$$

where $T^{*} \cap Q_{j}=\left\{q \in T^{*} \mid q \in Q_{j,(q)}\right\}$ and $(f, g)$ denotes the intersection multiplicity.

Proof. As shown before, the multiplicity of the strict transform at $q \in T^{*}$ of a generic element $g \in J$ fulfills $\mathrm{mt}\left(g_{(q)}, q\right)=\mathrm{mt}(X, q)=m_{q}$. In particular, we obtain for each branch $Q_{j}$ of $(C, z)$

$$
\begin{aligned}
\left(f, Q_{j}\right) \geq\left(g, Q_{j}\right) & \geq \sum_{q \in T^{*} \cap Q_{j}} m_{q} \cdot \operatorname{mt}\left(Q_{j,(q)}, q\right) \\
& =\sum_{q \in T^{*} \cap Q_{j}} \sum_{i=1}^{r} \operatorname{mt}\left(Q_{i,(q)}, q\right) \cdot \operatorname{mt}\left(Q_{j,(q)}, q\right) \\
& =2 \delta\left(Q_{j}\right)+\sum_{i \neq j}\left(Q_{i}, Q_{j}\right)+\sum_{q \in T^{*} \cap Q_{j}} \operatorname{mt}\left(Q_{j,(q)}, q\right)=: \alpha_{j} .
\end{aligned}
$$

Hence, we have the inclusion $J \subset J_{1}:=\bigcap_{j=1}^{r}\left\{f \in \mathcal{O}_{S, z} \mid\left(f, Q_{j}\right) \geq \alpha_{j}\right\}$. We can consider both as ideals in $\mathcal{O}_{C, z}$ and have to show that $\operatorname{dim}_{K}\left(\mathcal{O}_{C, z} / J_{1}\right)=\operatorname{deg}(X)$. To do so, let $n$ denote the injection

$$
n: \mathcal{O}_{C, z} \hookrightarrow \prod_{j=1}^{r} \mathbf{C}\left\{t_{j}\right\}=: \overline{\mathcal{O}}
$$

induced by a parametrization of $(C, z)$ and consider the image $n\left(J_{1}\right) \subset \overline{\mathcal{O}}$. For an element $f \in \overline{\mathcal{O}}$ the conditions on the intersection multiplicities $\left(f, Q_{j}\right)$ read as $f \in \prod_{j=1}^{r} t_{j}^{\alpha_{j}} \cdot \mathbf{C}\left\{t_{j}\right\}$. Hence

$$
\begin{aligned}
\operatorname{dim}_{K}\left(\mathcal{O}_{C, z} / J_{1}\right) & \geq \operatorname{dim}_{K}\left(\overline{\mathcal{O}} / \prod_{j=1}^{r} t_{j}^{\alpha_{j}} \cdot \mathbf{C}\left\{t_{j}\right\}\right)-\operatorname{dim}_{K}\left(\overline{\mathcal{O}} / \mathcal{O}_{C, z}\right) \\
& =\sum_{j=1}^{r} \alpha_{j}-\delta(C, z)=\operatorname{deg}(X) .
\end{aligned}
$$


Definition 2.9. Two generalized singularities $X_{0}, X_{1} \in \mathcal{G S}$, centred at $z$, are called isomorphic, $X_{0} \cong X_{1}$, if they are isomorphic as subschemes of $S$. $X_{0}$ and $X_{1}$ are called equivalent, $X_{0} \sim X_{1}$, if there exist germs (respectively multigerms, if the $X_{i}$ are reducible) $\left(C_{0}, z\right)$ defining $X_{0}$ and $\left(C_{1}, z\right)$ defining $X_{1}$, and a $T^{*}$-equimultiple family over some (reduced) open connected subset $T$ of $\mathbf{A}^{1}$ having $\left(C_{0}, T_{0}^{*}\right)$ and $\left(C_{1}, T_{1}^{*}\right)$ as fibres. Here, by a $T^{*}-$ equimultiple family over a (reduced) algebraic $k$-scheme $T$ we denote a flat family with section $\sigma$,

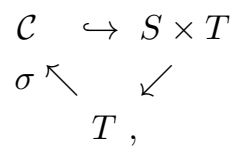

of reduced plane curve singularities $\left(\mathcal{C}_{t}, \sigma(t)\right) \subset S=S \times\{t\}$ which admits a simultaneous, embedded resolution, together with sections $\sigma_{q}$ through infinitely near points, defining a family $\mathcal{T}^{*}$ of trees $T_{t}^{*}, T^{*}\left(\mathcal{C}_{t}, \sigma(t)\right) \subset T_{t}^{*} \subset T\left(\mathcal{C}_{t}, \sigma(t)\right)$ such that the total transform of $\mathcal{C}$ is equimultiple along $\sigma_{q}, \sigma_{q}(t) \in T_{t}^{*}$. We denote such a family by $\left(\mathcal{C}, \mathcal{T}^{*}\right)$.

Since any $X \in \mathcal{G S}$ is defined by a generic element in $J_{X} \subset \mathcal{O}_{S, z}$, isomorphic schemes $X_{0}, X_{1}$ are defined by isomorphic germs which can be connected by a family of isomorphisms. Hence, $X_{0} \cong X_{1}$ implies $X_{0} \sim X_{1}$.

Definition 2.10. Let $X \in \mathcal{G S}$ with centre $z$ and $L=(L, z)$ be a smooth (multi)germ. Define $X \cap L$ to be the scheme-theoretic intersection. Set

$$
\begin{aligned}
T_{X}^{*} \cap L & :=\left\{q \in T_{X}^{*} \mid q \in L_{(q)}\right\}, \\
J_{X}: L & :=\left\{f \in \mathcal{O}_{S, z} \mid f L \in J_{X}\right\}, \\
X & : L:=Z\left(J_{X}: L\right) .
\end{aligned}
$$

We call $X: L$ the reduction of $\mathbf{X}$ by $L$.

Proposition 2.11. Let $X=X\left(C, T^{*}\right) \in \mathcal{G S}$ and $L \subset S$ be smooth at $z$, the centre of $X$.

(i) The reduction $X: L$ is a generalized singularity centred at $z$ and its tree $T_{X: L}^{*}=T^{*}: L$ is a subtree of $T^{*}$.

(ii) $\operatorname{mt}(X, q)-1 \leq \mathrm{mt}(X: L, q) \leq \mathrm{mt}(X, q)$ for all $q \in T^{*}$.

(iii) $\operatorname{deg}(X: L)=\operatorname{deg}(X)-\operatorname{deg}(X \cap L), \operatorname{deg}(X \cap L)=\sum_{q \in T^{*} \cap L} \operatorname{mt}(X, q)$.

(iv) Let $L$ be a line in $\mathbf{P}^{2}$, then there exists an exact sequence of ideal sheaves on $\mathbf{P}^{2}$

$$
0 \longrightarrow \mathcal{J}_{X: L / \mathbf{P}^{2}}(d-1) \stackrel{\cdot L}{\longrightarrow} \mathcal{J}_{X / \mathbf{P}^{2}}(d) \longrightarrow \mathcal{J}_{X \cap L / L}(d) \longrightarrow 0 .
$$

Proof. (iv) is obvious and (iii) follows from (iv), respectively the fact that

$$
\operatorname{deg}(X \cap L)=\operatorname{mt}(C \cap L, z)=\sum_{q \in T^{*} \cap L} \operatorname{mt}(X, q) .
$$


(i) will be proved by induction on $\operatorname{deg}(X)$. Again, we may begin with $\operatorname{deg}(X)=0$, which implies $X=\emptyset, T^{*}=\emptyset$ and $X: L=\emptyset, T^{*}: L=\emptyset$.

If $\operatorname{deg}(X)>0$ then $z \in T^{*}$ and we consider the blowing-up $\pi: \widehat{S} \longrightarrow S$ of $z$. By Lemma 2.6 the strict transform $C^{*}$ of $C$ fulfills $\operatorname{deg}\left(X\left(C^{*}, T^{*} \backslash\{z\}\right)\right)<\operatorname{deg}(X)$, hence by the induction assumption there exists a (multi)germ $D^{*}$ and a subtree $T_{D^{*}} \subset T^{*} \backslash\{z\}$ such that

$$
\begin{gathered}
J\left(C^{*}, T^{*} \backslash\{z\}\right): L^{*}=J\left(D^{*}, T_{D^{*}}\right) \\
T^{*}\left(D^{*}\right) \subset T_{D^{*}} \subset T\left(D^{*}\right),
\end{gathered}
$$

moreover, we can choose $D^{*}$ generically in $J\left(C^{*}, T^{*} \backslash\{z\}\right): L^{*}$ (such that $\operatorname{mt}\left(D^{*}\right)$ and the intersection multiplicity $\left(D^{*}, E\right)$ are minimal).

Blowing down $D^{*}$ we obtain a germ $D$ at $z$. Let $m:=\operatorname{mt}(C, z)$.

Case 1: $\operatorname{mt}(D, z)=m-a<m$.

Define the germ $C^{\prime}$ at $z$ by $C^{\prime}:=D \cdot L_{1} \cdot \ldots \cdot L_{a-1}$, where $L_{1}, \ldots, L_{a-1}$ are smooth germs with generic tangent directions at $z$. Then

$$
\begin{aligned}
f \in J\left(C, T^{*}\right): L & \Leftrightarrow \operatorname{mt}(f, z) \geq m-1, L^{*} \hat{f}:(m-1) E \in J\left(C^{*}, T^{*} \backslash\{z\}\right) \\
& \Leftrightarrow \operatorname{mt}(f, z) \geq m-1, \hat{f}:(m-1) E \in J\left(D^{*}, T_{D^{*}}\right) \\
& \Leftrightarrow \operatorname{mt}(f, z) \geq m-1, \hat{f}:(m-1) E \in J\left(D^{*} \cdot L_{1}^{*} \ldots L_{a-1}^{*}, T_{D^{*}}\right) \\
& \Leftrightarrow f \in J\left(C^{\prime}, T^{*}\right)
\end{aligned}
$$

with $T^{* *}:=T_{D^{*}} \cup\{z\}$.

Case 2: $\operatorname{mt}(D, z)=m$.

By the induction assumption, there exists a (multi)germ $\bar{D}^{*}$ and a subtree $T_{\bar{D}^{*}} \subset T_{D^{*}} \subset T^{*} \backslash\{z\}$ such that

$$
\begin{gathered}
J\left(D^{*}, T_{D^{*}}\right): E=J\left(\bar{D}^{*}, T_{\bar{D}^{*}}\right) \\
T^{*}\left(\bar{D}^{*}\right) \subset T_{\bar{D}^{*}} \subset T\left(\bar{D}^{*}\right) .
\end{gathered}
$$

We choose $\bar{D}^{*}$ generically in the ideal $J\left(D^{*}, T_{D^{*}}\right): E$. Since $D^{*} \in J\left(\bar{D}^{*}, T_{\bar{D}^{*}}\right)$, $p:=\left(D^{*}, E\right)-\left(\bar{D}^{*}, E\right) \geq 0$, and we define $C^{\prime}:=\bar{D} \cdot L_{1} \cdot \ldots \cdot L_{p}$, where $L_{1}, \ldots, L_{p}$ denote generic smooth germs at $z$ and $\bar{D}$ is the blowing-down of $\bar{D}^{*}$. Again

$$
f \in J\left(C, T^{*}\right): L \Leftrightarrow \operatorname{mt}(f, z) \geq m-1, L^{*} \hat{f}:(m-1) E \in J\left(C^{*}, T^{*} \backslash\{z\}\right) .
$$

Assuming $\mathrm{mt}(f, z)=m-1$ and $L^{*} \hat{f}:(m-1) E \in J\left(C^{*}, T^{*} \backslash\{z\}\right)$ we would have $f^{*} \in J\left(C^{*}, T^{*} \backslash\{z\}\right): L^{*}=J\left(D^{*}, T_{D^{*}}\right)$, hence $m-1=\left(f^{*}, E\right) \geq\left(D^{*}, E\right)=m$. Thus

$$
\begin{aligned}
f \in J\left(C, T^{*}\right): L & \Leftrightarrow \operatorname{mt}(f, z) \geq m, \hat{f}: m E \in J\left(D^{*}, T_{D^{*}}\right): E=J\left(\bar{D}^{*}, T_{\bar{D}^{*}}\right) \\
& \Leftrightarrow \operatorname{mt}(f, z) \geq m, \hat{f}: m E \in J\left(\bar{D}^{*} \cdot L_{1}^{*} \cdot \ldots \cdot L_{p}^{*}, T_{\bar{D}^{*}}\right) \\
& \Leftrightarrow f \in J\left(C^{\prime}, T^{* *}:=T_{\bar{D}^{*}} \cup\{z\}\right) .
\end{aligned}
$$

Note that in this case we have $\mathrm{mt}\left(C^{\prime}, z\right)=\mathrm{mt}(C, z)$, while in the first case we had $\mathrm{mt}\left(C^{\prime}, z\right)=\mathrm{mt}(C, z)-1$. This implies (ii). 
Examples. 1. Let $(C, z)$ be a node, $C=y^{2}-x^{2}$ with respect to local coordinates $(x, y)$ at $z, T^{*}=T^{*}(C, z)=\{z\}$, then for each $L$ the reduction $X: L$ is the generalized singularity given by a smooth germ at $z$ and the tree $T_{X: L}^{*}=T^{*}=\{z\}$.

2. In the case of an $A_{2 k-1}$-singularity $(k \geq 2) C=y^{2}-x^{2 k}$ with the tree of essential points $T^{*}=\left\{z=q_{0}, \ldots, q_{k-1}\right\}$ we have $J_{X}=\left\langle y^{2}, y x^{k}, x^{2 k}\right\rangle$. If $L=y$, then $J_{X: L}=\left\langle y, x^{k}\right\rangle$ and $X: L$ is the generalized singularity given by the smooth germ $y-x^{k}$ and the tree $T_{X: L}^{*}=\left\{z=q_{0}, \ldots, q_{k-1}\right\}$. On the other hand, if $L=x$, then $J_{X: L}=\left\langle y^{2}, y x^{k-1}, x^{2 k-1}\right\rangle$ and $X: L$ is the generalized singularity scheme given by $\left(y-x^{k}\right)\left(y+x^{k-1}\right)$ and the tree $T_{X: L}^{*}=T^{*}$.

3. For an $A_{2 k}$-singularity $C=y^{2}-x^{2 k+1}$ with the tree of essential points $T^{*}=\left\{z=q_{0}, \ldots, q_{k+1}\right\}$ we have $J_{X}=\left\langle y^{2}, y x^{k+1}, x^{2 k+1}\right\rangle$. If $L=y$, then $J_{X: L}=\left\langle y, x^{k+1}\right\rangle$ and $X: L$ is the generalized singularity given by the smooth germ $y-x^{k+1}$ and the tree $T_{X: L}^{*}=\left\{z=q_{0}, \ldots, q_{k}\right\}$. On the other hand, if $L=x$, then $J_{X: L}=\left\langle y^{2}, y x^{k}, x^{2 k}\right\rangle$ and $X: L$ is the singularity scheme given by an $A_{2 k-1}$-singularity.

Definition 2.12. Denote by $\mathcal{G} \mathcal{S}_{1} \subset \mathcal{G S}$ the subclass of such $X$ defined by germs $(C, z)$ with all branches smooth.

Lemma 2.13. The class $\mathcal{G S}_{1}$ is closed with respect to the equivalence relation $\sim$ and with respect to reduction by $L$.

Proof. The first statement is obvious, the second is a consequence of the proof of 2.11.

Lemma 2.14. Let $X=X\left(C, T^{*}\right) \in \mathcal{G S}$ be non-empty and $L$ smooth at $z$.

(i) There exists a branch $Q$ of $(C, z)$ such that $T^{*} \cap L \subset T^{*} \cap Q$.

(ii) If $Q$ is a non-singular branch of $(C, z)$ and if $M \subset T^{*}$ is a connected subtree with $T^{*} \cap L \subset M \subset T^{*} \cap Q$ then there exists $X_{1}=X\left(C_{1}, T_{1}^{*}\right) \in \mathcal{G S}$, $X_{1} \cong X, Q_{1}$ a smooth branch of $\left(C_{1}, z\right) \cong(C, z), M \cong M_{1} \subset Q_{1} \cap T_{1}^{*}$ such that $T_{1}^{*} \cap L=M_{1}$.

Proof. (i) is obvious. For the proof of (ii), we may assume that $z \in L \cap T^{*}$. We choose coordinates $(x, y)$ at $\mathrm{z}$ such that $L=y$ and the non-singular branch $Q$ is given by $y-\sum_{i=1}^{\infty} \alpha_{i} x^{i}$. Let $N:=\#(M)$ and $f$ be the power series defining $(C, z)$. The germs

$$
C_{t}=f\left(x, y+t \cdot\left(\sum_{i=1}^{N-1} \alpha_{i} x^{i}+\beta x^{N}\right)\right), \beta \in K \text { generic, }
$$

define an equianalytic family such that $\left(C_{0}, z\right)=(C, z)$ and $\left(C_{1}, z\right)$ has a branch $Q_{1}$ given by $y-\sum_{i \geq N} \tilde{\alpha}_{i} x^{i}$. Especially for the corresponding trees $T_{1}^{*} \cong T^{*}$, respectively $M_{1} \cong M$, we have $M_{1} \subset Q_{1} \cap T_{1}^{*}$ and, since $\beta$ was chosen generically,

$$
\#\left(T_{1}^{*} \cap L\right)=\#\left(T_{1}^{*} \cap L \cap Q_{1}\right)=N=\#\left(M_{1}\right),
$$


hence $T_{1}^{*} \cap L=M_{1}$.

Lemma 2.15. Let $X=X\left(C, T^{*}\right) \in \mathcal{G S}, Q$ be a smooth branch of $(C, z)$ and $L$ be smooth at $z$.

(i) If $T^{*} \cap Q \subset T^{*} \cap L$ then $\operatorname{mt}(X: L)=\mathrm{mt} X-1$.

(ii) If $T^{*} \cap Q=T^{*} \cap L$ then $X: L$ is defined by the germ $\left(C^{\prime}, z\right)$ and the tree $T^{*} \cap T\left(C^{\prime}, z\right)$, where $C^{\prime}$ is a factor of $C$ such that $C=C^{\prime} Q$.

Proof. Let $C=C^{\prime} \cdot Q$ at $z$. Then, obviously $C^{\prime} \in J_{X}: L$, hence the multiplicity of a generic element is at most $\mathrm{mt} X-1$. Thus, (i) follows from 2.11 (ii).

If $T^{*} \cap Q=T^{*} \cap L$, then we have for each $q \in T^{*}$

$$
\operatorname{mt}\left(\widehat{C}_{(q)}^{\prime}, q\right)=\operatorname{mt}\left(\widehat{C}_{(q)}, q\right)-\operatorname{mt}\left(\widehat{L}_{(q)}, q\right),
$$

which implies (ii).

Lemma 2.16. Let $X=X\left(C, T^{*}\right) \in \mathcal{G S}$ and $(L, z)$ be a smooth germ. Then we have $\operatorname{deg}((X: L) \cap L) \leq \operatorname{deg}(X \cap L)$. Moreover,

(i) if $\operatorname{deg}((X: L) \cap L)=\operatorname{deg}(X \cap L)$ then $\operatorname{mt}(X: L)=\operatorname{mt} X$;

(ii) if $\operatorname{deg}((X: L) \cap L))<\operatorname{deg}(X \cap L)$ then either $\operatorname{mt}(X: L)<\operatorname{mt} X$ or the defining germ $\left(C^{\prime}, z\right)$ of $X: L$ has a branch $Q^{\prime}$ satisfying $T_{X: L}^{*} \cap Q^{\prime} \subset T_{X: L}^{*} \cap L$. In any case, $\operatorname{mt}\left(X: L^{2}\right) \leq \mathrm{mt} X-1$.

Proof. By Prop. 2.11 (ii), $\mathrm{mt}(X: L, q) \leq \mathrm{mt}(X, q)$ for all $q \in T^{*}$, hence the inequality. Therefore, $\operatorname{deg}((X: L) \cap L)=\operatorname{deg}(X \cap L)$ implies $\operatorname{mt}(X: L, q)=\operatorname{mt}(X, q)$ for any $q \in T^{*} \cap L$, in particular for $q=z$. This implies (i).

Let $\operatorname{deg}((X: L) \cap L)<\operatorname{deg}(X \cap L)$ and $T^{*} \cap L=\left\{z=q_{0}, q_{1}, \ldots, q_{\ell}\right\}$. Recall the construction of $X: L=X\left(C^{\prime}, T^{*}\right)$ in the proof of Proposition 2.11 (i). In Case 1 we had

$$
\operatorname{mt}(X: L)=\operatorname{mt}\left(C^{\prime}, z\right)=\operatorname{mt}(C, z)-1=\operatorname{mt} X-1 .
$$

In Case $2, C^{\prime}$ was given as

$$
C^{\prime}=\bar{D} \cdot L_{1} \cdot \ldots \cdot L_{p} \text { with } p=\left(C^{*}, E\right)-\left(\bar{D}^{*}, E\right) .
$$

Assume that there is no branch $Q^{\prime}$ of $C^{\prime}$ such that $T^{* *} \cap Q^{\prime} \subset T^{* *} \cap L$, in particular, $p=0$. Then $\bar{D}^{*}=C^{*}$, the strict transform of $C^{\prime}$, and

$$
\operatorname{mt}\left(C_{\left(q_{0}\right)}\right)=\left(C^{*}, E\right)=\left(C^{* *}, E\right)=\operatorname{mt}\left(C_{\left(q_{0}\right)}^{\prime}\right) .
$$

On the other hand, the intersection multiplicity $\left(C^{*}, E\right)$ is just the sum of all mt $\left(C_{(q)}^{\prime}\right)$ with $q \in T\left(C^{\prime}\right) \cap E_{(q)}$. By the above assumption all those $q$ are essential for $C^{\prime}$, which implies

$$
\sum_{q \in T^{*}\left(C^{\prime}\right) \cap E_{(q)}} \operatorname{mt}\left(C_{(q)}^{\prime}\right)=\left(C^{*}, E\right)=\left(C^{*}, E\right)=\sum_{q \in T(C) \cap E_{(q)}} \operatorname{mt}\left(C_{(q)}\right) .
$$

Since $T^{*}\left(C^{\prime}\right) \subset T^{*}(C)$, it follows $\operatorname{mt}\left(C_{(q)}^{\prime}\right)=\operatorname{mt}\left(C_{(q)}\right)$ for all $q \in T^{*}\left(C^{\prime}\right) \cap E_{(q)}$, especially $\mathrm{mt}\left(C_{\left(q_{1}\right)}^{\prime}\right)=\operatorname{mt}\left(C_{\left(q_{1}\right)}\right)$. By induction, we obtain $\operatorname{mt}\left(C_{\left(q_{i}\right)}^{\prime}\right)=\operatorname{mt}\left(C_{\left(q_{i}\right)}\right)$ for each $i \in\{0, \ldots, \ell\}$, which is impossible (cf. Proposition 2.11 (iii)). 
Definition 2.17. Given a $T^{*}$-equimultiple family of plane curve singularities $\left(\mathcal{C}, \mathcal{T}^{*}\right)$ over a reduced algebraic $K$-scheme $T$ (as defined in 2.9), we define

$\mathfrak{J}\left(\mathcal{C}, \mathcal{T}^{*}\right):=\left\{f \in \mathcal{O}_{S \times T, \sigma(T)} \mid \operatorname{mt}\left(\hat{f}_{\sigma_{q}(t)}, \sigma_{q}(t)\right) \geq \operatorname{mt}\left(\hat{\mathcal{C}}_{\sigma_{q}(t)}, \sigma_{q}(t)\right)\right.$ for $\left.q \in T_{t}^{*}, t \in T\right\}$

and

$$
\mathcal{X}\left(\mathcal{C}, \mathcal{T}^{*}\right):=\mathcal{O}_{S \times T, \sigma(T)} / \mathfrak{J}\left(\mathcal{C}, \mathcal{T}^{*}\right)
$$

A flat family $\mathcal{X}$ of fat points in $S \times T$ is called a family of generalized singularity schemes, if $\mathcal{X}=\mathcal{X}\left(\mathcal{C}, \mathcal{T}^{*}\right)$ for some $T^{*}$-equimultiple family $\left(\mathcal{C}, \mathcal{T}^{*}\right)$.

Since we consider only reduced base spaces $T$, then flatness just means that the total length is constant, which holds for a family $\mathcal{X}\left(\mathcal{C}, \mathcal{T}^{*}\right)$ by Lemma 2.6. It is easily seen that the functor

$\underline{\mathcal{G S}}: T \mapsto\{$ families of generalized singularity schemes over $T\}$

is representable by a locally closed subscheme $G S$ of the punctual Hilbert scheme of $S$.

Proposition 2.18. Let $X \in \mathcal{G S}, L$ be smooth and $Y=X: L$. For almost all $Y^{\prime} \sim Y$ satisfying $\operatorname{deg}\left(Y^{\prime} \cap L\right)=\operatorname{deg}(Y \cap L)$ there exists a generalized singularity scheme $X^{\prime} \sim X$ such that $\operatorname{deg}\left(X^{\prime} \cap L\right)=\operatorname{deg}(X \cap L)$ and $Y^{\prime}=X^{\prime}: L$.

Proof. M Let $\mathcal{X}=\mathcal{X}\left(\mathcal{C}, \mathcal{T}^{*}\right)$ be a family of generalized singularity schemes over the reduced base space $T, t \in T$. The construction of $X: L$ given in the proof of 2.11 shows that we can simultaneously reduce the fibres of $\mathcal{X}$ by $L$. Hence we have a natural transformation

$$
\rho_{L}: \underline{\mathcal{G S}} \longrightarrow \underline{\mathcal{G S}}, \mathcal{X} \mapsto \mathcal{X}: L
$$

inducing a morphism $\rho_{L}: G S \longrightarrow G S$. Notice that two generalized singularity schemes $X_{1}, X_{2}$ are equivalent if and only if they are in the same connected component of $G S$. Therefore, to prove the proposition, it is enough to show that the restriction $\rho_{L, X}: G S_{L, X} \longrightarrow G S_{L, Y}$ of $\rho_{L}$ to the connected component $G S_{L, X}$ of $\left\{X^{\prime} \in G S \mid \operatorname{deg}\left(X^{\prime} \cap L\right)=\operatorname{deg}(X \cap L)\right\}$ containing $X$ is dominant. But this follows immediately from the fact that the dimension of the fibre $\rho_{L, X}^{-1}(Y)$ is just $\#\left(T_{X}^{*}\right)-\left(\#\left(T_{Y}^{*}\right)+\#\left(T_{X}^{*} \cap L\right)-\#\left(T_{Y}^{*} \cap L\right)\right)=\operatorname{dim}\left(G S_{L, X}\right)-\operatorname{dim}\left(G S_{L, Y}\right)$.

In the following, we shall introduce the second basic operation on generalized singularities, the extension. For this, it is convenient to work with the field $K\{\{x\}\}$ of fractional power series

$$
\sum_{i=0}^{\infty} \alpha_{i} x^{i / n}, \alpha_{i} \in K, n \in \mathbf{N} .
$$

\footnotetext{
${ }^{1}$ We should like to thank I. Tyomkin for an idea leading to the present proof.
} 
Any germ $(C, z)$ of a reduced curve singularity may be given, with respect to suitable local coordinates $x, y$, as

$$
C=\prod_{i=1}^{m}\left(y-\xi_{i}(x)\right), \quad m=\operatorname{mt}(C, z), \quad \xi_{1}, \ldots, \xi_{m} \in K\{\{x\}\} .
$$

Moreover, if $(C, z)$ is irreducible and $t=x^{1 / m}$, then

$$
C=\prod_{i=1}^{m}\left(y-\xi\left(\eta^{i} t\right)\right), \quad \xi \in K[[t]],
$$

with $\eta$ a primitive $m$-th root of unity. We define the intersection multiplicity of two fractional power series $\xi_{i}, \xi_{j} \in K\{\{x\}\}$ to be

$$
\left(\xi_{i}, \xi_{j}\right):=\max \left\{\rho \in \mathbf{Q} \mid x^{\rho} \text { divides } \xi_{i}(x)-\xi_{j}(x)\right\} .
$$

Lemma 2.19. Let $X=X\left(C, T^{*}(C, z)\right) \in \mathcal{S}$ be a singularity scheme and $(C, z)$ given as above. Then

$$
\operatorname{deg}(X)=\sum_{1 \leq i<j \leq m}\left(\xi_{i}, \xi_{j}\right)+\sum_{i=1}^{m} \max _{j \neq i}\left(\xi_{i}, \xi_{j}\right)+\frac{m-r}{2},
$$

where $m=\mathrm{mt}(C, z)$ and $r$ is the number of branches of $(C, z)$.

Proof. It is well-known that the intersection multiplicity at $z$ of the polar $P_{q}(C)$ $(q=(0: 1: 0))$ given by the power series

$$
\frac{\partial C}{\partial y}=\sum_{i=1}^{m} \prod_{j \neq i}\left(y-\xi_{j}(x)\right)
$$

and the curve $\mathrm{C}$ fulfills $\sum_{i \neq j}\left(\xi_{i}, \xi_{j}\right)=\operatorname{mt}\left(P_{q}(C) \cap C, z\right)=2 \delta(C, z)+m-r$. Hence, by Lemma 2.6, it suffices to show

$$
\sum_{q \in T^{*}(C)} m_{q}=\sum_{i=1}^{m} \max _{j \neq i}\left(\xi_{i}, \xi_{j}\right)+m-r .
$$

In the case of an irreducible germ $(C, z)$ it follows from the above description of the $\xi_{i}$ that the numbers $\left(\xi_{i}, \xi_{j}\right)$ do only depend on the characteristic terms of the Puiseux expansion, and the statement is an immediate consequence of the algorithm to compute the multiplicity sequence from the Puiseux pairs (cf. [BrK]).

In the case of a reducible germ $(C, z)$, we have to investigate, additionally, the case of two branches $Q_{k}=\prod_{i=1}^{m_{k}}\left(y-\xi^{(k)}\left(\eta_{k}^{i} t\right)\right)(k \in\{1,2\})$ such that $T\left(Q_{1}\right) \cap T\left(Q_{2}\right)$ contains a non-essential point $q$ of $T\left(Q_{1}\right)$ and for all branches $Q \neq Q_{1}$ of $(C, z)$ and all successors $\hat{q}$ of $q$ in $T\left(Q_{1}\right)$ we have $\hat{q} \notin T(Q)$. In this case, obviously, $m_{2}=M m_{1}, M \in \mathbf{N}$, and we can assume the maximum intersection multiplicity of the fractional power series $\xi_{i}^{(1)}(x)=\xi^{(1)}\left(\eta^{M i} x^{1 / m_{1}}\right)$ ( $\eta$ a primitive $m_{2}$-th root of unity, $\left.i \in\left\{1, \ldots, m_{1}\right\}\right)$ with any other fractional power series in the equation of 
$(C, z)$ to be realized by $\xi_{M i}^{(2)}(x)=\xi^{(2)}\left(\eta^{M i} x^{1 / m_{2}}\right)$. Then we have

$$
\begin{aligned}
\sum_{q \in T^{*}(C)} m_{q}\left(Q_{1}\right) & =\frac{1}{M} \sum_{q \in T^{*}(C)} m_{q}\left(Q_{1}\right) m_{q}\left(Q_{2}\right)-\sum_{q \in T^{*}(C)} m_{q}\left(Q_{1}\right)\left(m_{q}\left(Q_{1}\right)-1\right) \\
& =\frac{1}{M} \cdot \operatorname{mt}\left(Q_{1} \cap Q_{2}, z\right)-2 \cdot \delta\left(Q_{1}\right) \\
& =\sum_{i=1}^{m_{1}} \sum_{j=1}^{m_{1}}\left(\xi_{i}^{(1)}, \xi_{M j}^{(2)}\right)-\sum_{i \neq j}\left(\xi_{i}^{(1)}, \xi_{j}^{(1)}\right)+m_{1}-1,
\end{aligned}
$$

and the statement follows from the fact that for $i \neq j$ the intersection multiplicities $\left(\xi_{i}^{(1)}, \xi_{j}^{(1)}\right)$ and $\left(\xi_{i}^{(1)}, \xi_{M j}^{(2)}\right)$ coincide.

Lemma 2.20. Let $X=X\left(C, T^{*}\right) \in \mathcal{G S}, L$ be smooth at $z$ and $q \in T^{*} \cap L \backslash\{z\}$. Let

$$
C=\prod_{i=1}^{n}\left(y-\xi_{i}(x)\right) \prod_{i=n+1}^{m}\left(y-\xi_{i}(x)\right), \quad m=\operatorname{mt}(C, z), \quad \xi_{i} \in K\{\{x\}\},
$$

be decomposed so that $\xi_{1}, \ldots, \xi_{n}$ are all fractional power series belonging to branches $Q$ of $(C, z)$ with $q \in Q_{(q)}$. Then there exists an integer $k \geq 0$ such that

$$
\begin{aligned}
& k<\left(\xi_{i}, \xi_{j}\right) \text { for } 1 \leq i<j \leq n, \\
& k \geq\left(\xi_{i}, \xi_{j}\right) \text { for } 1 \leq i \leq n<j \leq m .
\end{aligned}
$$

More precisely, if $L=y$, then

$$
\xi_{i}(x)=\sum_{\rho \geq 0} \alpha_{\rho}^{(i)} x^{\rho}, \quad \alpha_{\rho}^{(i)} \in K, \rho \in \mathbf{Q}
$$

belongs to $Q$ with $q \in Q_{(q)}$ if and only if $\alpha_{\rho}^{(i)}=0$ for $\rho \leq k$.

Proof. Let $L=y$ and $T^{*} \cap L=\left\{z=q_{0}, q_{1}, \ldots, q_{\ell}\right\}$. Moreover, let the branch $Q$ be given by

$$
Q=y^{p}+a_{1}(x) y^{p-1}+\cdots+a_{p}(x)=\prod_{i=1}^{p}\left(y-\xi\left(\eta^{i} x^{1 / p}\right)\right),
$$

where $\xi(t)=\sum_{j=0}^{\infty} \alpha_{j} t^{j} \in K[[t]]$. To prove the lemma, it is sufficient to show that for each $k \in\{1, \ldots, \ell\}$

$$
q_{k} \in Q_{\left(q_{k}\right)} \Leftrightarrow \alpha_{j}=0 \text { for each } j \leq k \cdot p .
$$

We proceed by induction on the length $k+1$ of the tree $\left\{q_{0}, \ldots, q_{k}\right\}$. Obviously, $z=q_{0} \in Q$ if and only if $a_{p}(0)=0$, that is, if and only if $\alpha_{0}=0$. Furthermore, the total transform of $Q$ at $q_{1}$ reads as $\widehat{Q}_{\left(q_{1}\right)}=\prod_{i=1}^{p}\left(u v-\xi\left(\eta^{i} u^{1 / p}\right)\right)$, hence $q_{1} \in Q_{\left(q_{1}\right)}$ if and only if $\xi(t)=\sum_{j=p+1}^{\infty} \alpha_{j} t^{j}$. Then $Q_{\left(q_{1}\right)}$ has the equation

$$
Q_{\left(q_{1}\right)}=\prod_{i=1}^{p}\left(v-\tilde{\xi}\left(\eta^{i} u^{1 / p}\right)\right)
$$

at $q_{1}$, where $\tilde{\xi}(t)=\sum_{j=1}^{\infty} \alpha_{j+p} t^{j}$, and we complete the proof by applying the induction hypothesis to $Q_{\left(q_{1}\right)}$ and the tree $\left\{q_{1}, \ldots, q_{k}\right\} \subset\left(T^{*} \backslash\{z\}\right) \cap L$. 
Definition 2.21. Using the notations and hypotheses of Lemma 2.20, let

$$
\xi_{i}(x)=\sum_{\rho>k} \alpha_{\rho}^{(i)} x^{\rho}, i=1, \ldots, n .
$$

Define a germ $(C(q), z)$ by

$$
C(q):=\prod_{i=1}^{n}\left(y-x \xi_{i}(x)\right) \prod_{i=n+1}^{m}\left(y-\xi_{i}(x)\right) .
$$

Call $C(q)$ the extension of $\mathbf{C}$ at $q$.

Lemma 2.22. The tree $T^{*}(C(q))$ of essential points of $C(q)$ has the following structure: insert in $T^{*}(C)$ a new point $q^{\prime}$ between $q$ and its predecessor $\bar{q}$. Moreover, $\operatorname{mt}\left(C(q)_{(p)}, p\right)=\operatorname{mt}\left(C_{(p)}, p\right)$ for all $p \in T^{*}(C(q)) \backslash\left\{q^{\prime}\right\}=T^{*}(C)$ and

$$
\operatorname{mt}\left(C(q)_{\left(q^{\prime}\right)}, q^{\prime}\right)=\sum_{Q: q \in Q_{(q)}} \operatorname{mt}\left(Q_{(\bar{q})}, \bar{q}\right) .
$$

Any tree $T^{*}$ containing $T^{*}(C)$ becomes extended by this operation to a tree $T^{*}(q)$. We call $T^{*}(q)$ the extension of $\mathbf{T}^{*}$ at $q$.

Proof. As in the proof of 2.20, let $T^{*} \cap L=\left\{z=q_{0}, q_{1}, \ldots, q_{\ell}\right\}$. An easy consideration shows that for $q=q_{k}$ the strict transform $C(q)_{(q)}$ of the extension of $C$ at $q$ has the local equation

$$
\prod_{i=1}^{n}\left(v-\frac{1}{u^{k-1}} \xi_{i}(u)\right)=\prod_{i=1}^{n}\left(v-\sum_{\rho>0} \alpha_{\rho+k}^{(i)} u^{\rho+1}\right)
$$

while $C(q)_{\left(q_{k+1}\right)}$ is given by

$$
\prod_{i=1}^{n}\left(v-\frac{1}{u^{k}} \xi_{i}(u)\right)=\prod_{i=1}^{n}\left(v-\sum_{\rho>0} \alpha_{\rho+k}^{(i)} u^{\rho}\right)
$$

which corresponds to the equation of $C_{(q)}$ at $q$. Hence, the structure of $T^{*}(C(q))$ can be described as in the lemma. Moreover, notice that for $i \leq k$

$$
\operatorname{mt}\left(C(q)_{\left(q_{i}\right)}, q_{i}\right)=n+\sum_{Q: q \notin Q_{(q)}} \operatorname{mt}\left(Q_{\left(q_{i}\right)}, q_{i}\right),
$$

which implies the statement about the multiplicities.

Definition 2.23. Let $X=X\left(C, T^{*}\right) \in \mathcal{G S}, L$ be a smooth germ at $z$ and $q \in$ $T^{*} \cap L \backslash\{z\}$. We define the extension of $\mathbf{X}$ at $q$ to be

$$
X(q):=X\left(C(q), T^{*}(q)\right) \in \mathcal{G S} .
$$

Examples. 1. Let $(C, z)$ be an ordinary cusp, $C=y^{2}-x^{3}$ with respect to local coordinates $(x, y)$ at $z, T^{*}=T^{*}(C, z)=\left\{z=q_{0}, q_{1}, q_{2}\right\}$ with (strict) multiplicities $m_{z}=2, m_{q_{1}}=m_{q_{2}}=1$; we write $T^{*}=\underset{2}{*}-\underset{1}{*}-\underset{1}{*}$.

Moreover, let $L=y$, that is $T^{*} \cap L=\left\{q_{0}, q_{1}\right\}$. The extension $X\left(q_{1}\right)$ is given by $y^{2}-x^{5}$ and the tree $T^{*}\left(q_{1}\right)=\underset{2}{*}-\underset{2}{*}-\underset{1}{*}-\underset{1}{*}$. 
2. Let $X \in \mathcal{S}$ be given by $C=\left(y^{2}-x^{3}\right)\left(y^{2}-x^{5}\right)$ and the tree of essential points

$$
T^{*}=\underset{4}{*}-\underset{3}{*}<\underset{1}{*} \underset{*}{*}-\stackrel{1}{*} .
$$

If $L=y$, then $T^{*} \cap L=\left\{z=q_{0}, q_{1}, q_{2}\right\}$ and the extension $X\left(q_{1}\right)$ is given by $\left(y^{2}-x^{5}\right)\left(y^{2}-x^{7}\right)$ and the tree

$$
T^{*}\left(q_{1}\right)=\underset{4}{*}-\underset{4}{*}-\underset{3}{*}<_{1}^{*}-\underset{*}{*},
$$

whence the extension $X\left(q_{2}\right)$ is given by $\left(y^{2}-x^{3}\right)\left(y^{2}-x^{7}\right)$ and

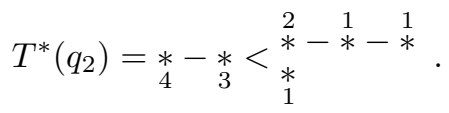

Lemma 2.24. With the notations of the preceding definition, assume that

$$
H^{1}\left(S, \mathcal{J}_{X(q) / \mathbf{P}^{2}}(d)\right)=0 .
$$

Then there exists an $X^{\prime} \in \mathcal{G S}, X^{\prime} \sim X$ such that $\operatorname{deg}\left(X^{\prime} \cap L\right)=\operatorname{deg}(X \cap L)$ and $H^{1}\left(S, \mathcal{J}_{X^{\prime} / \mathbf{P}^{2}}(d)\right)=0$.

Proof. Let $(x, y)$ be coordinates in a neighbourhood of $z=(0,0)$, such that $L=y$ and $(C, z)$ is given as in Lemma 2.20. For $t \in \mathbf{A}^{1}$ define

$$
C_{t}:=\underbrace{\prod_{i=1}^{n}\left(y-((1-t) x+t) \cdot \xi_{i}(x)\right)}_{C_{t}^{1}} \cdot \underbrace{\prod_{i=n+1}^{m}\left(y-\xi_{i}(x)\right)}_{C^{2}} .
$$

For $t \neq 0$ there is an obvious isomorphism $\varphi_{t}:\left(C_{t}^{1}, z\right) \stackrel{\cong}{\longrightarrow}\left(C_{1}^{1}, z\right)=\left(C^{1}, z\right) \subset(C, z)$ and for two branches $Q_{t}^{1}$ (resp. $\left.Q^{2}\right)$ of $\left(C_{t}^{1}, z\right)$ (resp. $\left(C^{2}, z\right)$ the intersection multiplicity at $z$ fulfills $\mathrm{mt}\left(Q_{t}^{1} \cap Q^{2}, z\right)=\operatorname{mt}\left(Q_{1}^{1} \cap Q^{2}, z\right)$, hence $\left(C_{t}, z\right) \sim(C, z)$. Moreover, for $t \neq 0$ sufficiently small, $C_{t}$ has an ordinary $n$-fold point at $z_{t}=$ $(-t /(1-t), 0)$.

Define $T_{t}^{*}$ as the union of $\left\{z_{t}\right\}$ with the tree $T^{2 *}$ corresponding to $C^{2}$ and the tree induced by $\varphi_{t}$ from $T^{1 *}$ (corresponding to $C^{1}$ ). $T_{t}^{*}$ is well-defined since $L \cap C_{t} \supset L \cap C$ for each $t \in \mathbf{A}^{1}$. Thus, we have defined a family $\mathcal{X}$ with fibres $X_{t}=X\left(C_{t}, T_{t}^{*}\right)$ centred at the multigerm $\left\{z, z_{t}\right\}$. Obviously $\mathcal{X}$ is flat in $t=0$ since for small $t \neq 0$ (by 2.6 and 2.22)

$$
\operatorname{deg}\left(X_{t}\right)=\operatorname{deg}(X)+\frac{n(n+1)}{2}=\operatorname{deg}(X(q))=\operatorname{deg}\left(X_{0}\right) .
$$

Hence, the family $\mathfrak{J}$ of ideals $J_{X_{t}}=J\left(C_{t}, T_{t}^{*}\right)$ is flat in $t=0$, which implies, by semicontinuity, the vanishing of $H^{1}\left(S, \mathcal{J}_{X_{t} / \mathbf{P}^{2}}(d)\right)$ for small $t \neq 0$.

Remark 2.25. The family $C_{t}$ of the above proof defines a deformation of the germ $(C(q), z)$ to $\left(C_{t},\left\{z, z_{t}\right\}\right)$, where $\left(C_{t}, z\right) \sim(C, z)$ and $\left(C_{t}, z_{t}\right)$ is an ordinary $n$-fold point, $n$ as in Lemma 2.22. In particular, $(C(q), z)$ is a degeneration of a germ which is topologically equivalent to $(C, z)$. 
3. $h^{1}$-VANISHING CRITERION FOR ZERO-DIMENSIONAL SCHEMES OF CLASS $\mathcal{G S}_{1}$ IN THE PLANE

Lemma 3.1. For any $d \geq 1$ and $X \in \mathcal{G S}_{1}$ satisfying

$$
\operatorname{deg} X<(3-2 \sqrt{2})(d-\operatorname{mt} X)^{2}
$$

there is $X^{\prime} \sim X$ with $h^{1}\left(\mathcal{J}_{X^{\prime} / \mathbf{P}^{2}}(d)\right)=0$.

Proof. We shall prove the following statement. Let $L \subset \mathbf{P}^{2}$ be a fixed straight line. There exist $\alpha, \beta \geq 0$ such that for any integer $d \geq 1$ and $X \in \mathcal{G S}_{1}$ satisfying

$$
\begin{aligned}
\operatorname{deg} X & \leq \beta(d-\operatorname{mt} X)^{2} \\
\operatorname{deg}(X \cap L) & \leq d-\alpha \frac{\operatorname{deg} X}{d}
\end{aligned}
$$

there exists $X^{\prime} \sim X$ with $\operatorname{deg}\left(X^{\prime} \cap L\right)=\operatorname{deg}(X \cap L), h^{1}\left(\mathcal{J}_{X^{\prime} / \mathbf{P}^{2}}(d)\right)=0$. Moreover, in Step 2, we show that for our approach the maximal possible value for $\beta$ is attained at

$$
\alpha=\sqrt{2}+1, \quad \beta=3-2 \sqrt{2} .
$$

Step 1. Assume that $X$ is an ordinary singularity, that means $T_{X}^{*}=\{z\}$. Then the ideal of $X$ in $\mathcal{O}_{\mathbf{P}^{2}, z}$ is defined by the vanishing of the coefficients of all monomials lying under the diagonal $[(0, \mathrm{mt} X),(\mathrm{mt} X, 0)]$ in the Newton diagram. Since mt $X<d$ by (3.3), these (linear) conditions are independent, hence $h^{1}\left(\mathcal{J}_{X / \mathbf{P}^{2}}(d)\right)=0$.

So, further on, we can suppose that $\operatorname{deg}(X)>0$ and that $X$ is not an ordinary singularity. We proceed by induction in $d$. For $d \leq 2$ there is nothing to consider. In the induction step, we reduce $X$ by $L$ and have to show

$$
\begin{gathered}
\operatorname{deg}(X: L)=\operatorname{deg} X-\operatorname{deg}(X \cap L) \leq \beta(d-1-\operatorname{mt}(X: L))^{2} \\
\operatorname{deg}((X: L) \cap L) \leq \operatorname{deg}(X \cap L) \leq d-1-\alpha \frac{\operatorname{deg}(X: L)}{d-1} .
\end{gathered}
$$

Then, by the induction assumption $h^{1}\left(\mathcal{J}_{Y / \mathbf{P}^{2}}(d-1)\right)=0$ for some $Y \sim X: L$, $\operatorname{deg}(Y \cap L)=\operatorname{deg}((X: L) \cap L)$. By Proposition 2.18 there exists $X^{\prime} \sim X$ with $X^{\prime}: L=Y$ and $\operatorname{deg}\left(X^{\prime} \cap L\right)=\operatorname{deg}(X \cap L)$. Since $h^{1}\left(\mathcal{J}_{X \cap L / L}(d)\right)=0$, because $\operatorname{deg}\left(X^{\prime} \cap L\right) \leq d-\alpha \cdot \operatorname{deg} X / d<d+1$, we obtain by Proposition 2.11 (iv) the desired relation $h^{1}\left(\mathcal{J}_{X^{\prime} / \mathbf{P}^{2}}(d)\right)=0$.

Step 2. Assume that

$$
\operatorname{deg}(X \cap L)=d-\alpha \frac{\operatorname{deg} X}{d} .
$$

Due to $\operatorname{mt}(X: L) \leq \mathrm{mt} X,(3.3)$ and (3.7), the first inequality in (3.6) will follow from

$$
\beta(d-\mathrm{mt} X)^{2}-d+\alpha \frac{\beta(d-\mathrm{mt} X)^{2}}{d} \leq \beta(d-1-\mathrm{mt} X)^{2},
$$


which is equivalent to

$$
d^{2}(1-\alpha \beta-2 \beta)+\alpha \beta(2 d-\mathrm{mt} X) \cdot \mathrm{mt} X+2 \beta d \cdot \mathrm{mt} X+\beta d \geq 0,
$$

hence, due to $\mathrm{mt} X \leq d$, it is enough to impose the condition

$$
1 \geq(\alpha+2) \beta .
$$

The second inequality in (3.6) will follow from

$$
\left(\alpha+\alpha^{2}\right) \cdot \operatorname{deg} X \leq(\alpha-1) d^{2}+d,
$$

which, by (3.3), holds true as

$$
\alpha-1 \geq \beta\left(\alpha+\alpha^{2}\right)
$$

We are interested in $\beta$ as large as possible. The inequality (3.9) gives

$$
\beta \leq \frac{\alpha-1}{\alpha+\alpha^{2}} \leq 3-2 \sqrt{2}
$$

and the maximal value is attained at $\alpha=\sqrt{2}+1$. So, from now on we suppose (3.5), especially the condition (3.8) is satisfied.

Step 3. Assume that

$$
\operatorname{deg}(X \cap L)<d-\alpha \frac{\operatorname{deg} X}{d},
$$

$X$ is not an ordinary singularity, there exists a branch $Q$ of $(C, z)$ such that $T_{X}^{*} \cap L=T_{X}^{*} \cap Q$, and there is no branch $Q^{\prime}$ of $(C, z)$ with $T_{X}^{*} \cap L \subsetneq T_{X}^{*} \cap Q^{\prime}$.

In this case $T_{X}^{*} \cap L$ consists of at least two points. Therefore $\operatorname{deg}(X: L)<\operatorname{deg} X$ and $\operatorname{deg}((X: L) \cap L) \leq \operatorname{deg}(X \cap L)-2$. Moreover, by (3.3) and (3.8), we have $\alpha \operatorname{deg} X / d \leq d-1$, hence

$$
\operatorname{deg}((X: L) \cap L) \leq(d-1)-\alpha \frac{\operatorname{deg}(X: L)}{d-1} .
$$

On the other hand, by Lemma 2.15, $\mathrm{mt}(X: L)=\mathrm{mt} X-1$, thus

$$
\operatorname{deg}(X: L)<\operatorname{deg} X \leq \beta(d-\operatorname{mt} X)^{2}=\beta(d-1-\operatorname{mt}(X: L))^{2} .
$$

Step 4. Assume that

$$
\operatorname{deg}(X \cap L)<d-\alpha \frac{\operatorname{deg} X}{d},
$$

and there is a branch $Q$ of $(C, z)$ such that: (1) $T_{X}^{*} \cap Q$ consists of points $z_{1}=z, \ldots, z_{r}$, naturally ordered, (2) $T_{X}^{*} \cap L$ consists of points $z_{1}, \ldots, z_{s}, 1 \leq s<r$, (3) the multiplicity $m=\operatorname{mt}\left(C_{\left(z_{s+1}\right)}, z_{s+1}\right)$ satisfies

$$
\operatorname{deg}(X \cap L)+m>d-\alpha \frac{\operatorname{deg} X}{d} .
$$

In this case, Lemma 2.6 gives

$$
\operatorname{deg} X>\frac{(\mathrm{mt} X)^{2}+m^{2}}{2} .
$$

Since $m \leq \mathrm{mt} X$ and due to (3.10), the first inequality in (3.6) will follow from

$$
\beta(d-\mathrm{mt} X)^{2}-d+\alpha \frac{\beta(d-\mathrm{mt} X)^{2}}{d}+\mathrm{mt} X \leq \beta(d-1-\mathrm{mt} X)^{2},
$$


or, equivalently, from

$$
(1-2 \beta-\alpha \beta)(d-\operatorname{mt} X)+\beta+\alpha \beta \frac{\operatorname{mt} X}{d}(d-\operatorname{mt} X) \geq 0,
$$

which holds true if (3.8) is satisfied. Similarly, the second inequality in (3.6) will follow from

$$
d-\alpha \frac{\operatorname{deg} X}{d} \leq d-1-\alpha \frac{\operatorname{deg} X-d+\alpha \operatorname{deg} X / d+m}{d-1}
$$

which, by (3.3), is satisfied, if

$$
(\alpha-1) d^{2}+d-\alpha m d \geq\left(\alpha+\alpha^{2}\right) \beta(d-\operatorname{mt} X)^{2} .
$$

The coefficient of $d^{2}$ is zero by (3.5), hence, it is enough to show that

$$
2 \beta\left(\alpha+\alpha^{2}\right) d \cdot \operatorname{mt} X-\beta\left(\alpha+\alpha^{2}\right)(\operatorname{mt} X)^{2}-\alpha m d \geq 0
$$

or, equivalently,

$$
m \leq \beta(1+\alpha)\left(2 \lambda-\frac{\lambda^{2}}{d}\right)=: \varphi(\lambda), \quad \lambda=\operatorname{mt} X .
$$

Indeed, due to (3.11) an (3.3), we have $\lambda \leq \frac{\sqrt{2 \beta}}{1+\sqrt{2 \beta}} d$ and

$$
m \leq \psi(\lambda):=\left\{\begin{array}{cl}
\lambda & \text { for } 0 \leq \lambda \leq \frac{\sqrt{\beta}}{1+\sqrt{\beta}} d \\
\sqrt{2 \beta(d-\lambda)^{2}-\lambda^{2}} & \text { for } \frac{\sqrt{\beta}}{1+\sqrt{\beta}} d \leq \lambda \leq \frac{\sqrt{2 \beta}}{1+\sqrt{2 \beta}} d .
\end{array}\right.
$$

Since $\varphi(d \sqrt{\beta} /(\sqrt{\beta}+1))=\psi(d \sqrt{\beta} /(\sqrt{\beta}+1))$ as $(3.5)$ holds, and $\varphi$ is increasing concavely in the segment $[0, d \sqrt{2 \beta} /(\sqrt{2 \beta}+1)]$, we obtain $m \leq \psi(\lambda) \leq \varphi(\lambda)$.

Step 5. Assume that

$$
\operatorname{deg}(X \cap L)<d-\alpha \frac{\operatorname{deg} X}{d},
$$

and that there is a (smooth) branch $Q$ of $(C, z)$ such that: (1) $T_{X}^{*} \cap Q$ consists of points $z_{1}=z, \ldots, z_{r}$, naturally ordered, (2) $T_{X}^{*} \cap L$ consists of points $z_{1}, \ldots, z_{s}$, $1 \leq s<r,(3)$ the multiplicity $m=\operatorname{mt}\left(C_{\left(z_{s+1}\right)}, z_{s+1}\right)$ satisfies

$$
\operatorname{deg}(X \cap L)+m \leq d-\alpha \frac{\operatorname{deg} X}{d} .
$$

Then by Lemma 2.14 we specialize the point $z_{s+1}$ on the line $L$ and consider the new scheme $\widetilde{X} \sim X$ with $\operatorname{deg}(\widetilde{X} \cap L)=\operatorname{deg}(X \cap L)+m$. By the semi-continuity of cohomology, $h^{1}\left(\mathcal{J}_{\widetilde{X} / \mathbf{P}^{2}}(d)\right)=0$ yields $h^{1}\left(\mathcal{J}_{X / \mathbf{P}^{2}}(d)\right)=0$. Thus, specializing points of $Q$ onto $L$ we come to one of the cases studied above. 


\section{4. $H^{1}$-Vanishing Criterion for Zero-Dimensional Schemes of Class $\mathcal{G} \mathcal{S}$}

IN THE PLANE

For a scheme $X \in \mathcal{G S}$ denote by $\mathrm{mt}_{s} X$ the sum of the multiplicities of all singular branches of the underlying germ $(C, z)$. Note that $\mathrm{mt} X, \mathrm{mt}_{s} X$ are invariant with respect to the extension (cf. 2.21)).

Lemma 4.1. For any integer $d \geq 1$ and any $X \in \mathcal{G S}$ satisfying

$$
\operatorname{deg} X \leq \beta_{0}\left(d-\operatorname{mt} X-\mathrm{mt}_{s} X\right)^{2},
$$

where $\beta_{0}=\left(\alpha_{0}+8\right)^{-1}=0.10340 \ldots$ and $\alpha_{0}=(31-3 \sqrt{85}) / 2=1.6706 \ldots$ is the positive root of the equation

$$
\left(\frac{\sqrt{4 \alpha^{3}+\alpha^{2}-4 \alpha}+\alpha-2}{2\left(1+\alpha+\alpha^{2}\right)}\right)^{2}=\frac{1}{\alpha+8},
$$

there is $X^{\prime} \sim X$ with $h^{1}\left(\mathcal{J}_{X^{\prime} / \mathbf{P}^{2}}(d)\right)=0$.

Proof. As in the proof of Lemma 3.1, we shall obtain a more general statement. Let $L \subset \mathbf{P}^{2}$ be a fixed straight line. There exist $\alpha, \beta>0$ such that, for any integer $d \geq 1$ and any $X \in \mathcal{G S}$, satisfying

$$
\operatorname{deg} X \leq \beta\left(d-\operatorname{mt} X-\mathrm{mt}_{s} X\right)^{2}, \quad \operatorname{deg}(X \cap L) \leq d-\alpha \frac{\operatorname{deg} X}{d},
$$

there exists $X^{\prime} \sim X$ with $h^{1}\left(\mathcal{J}_{X^{\prime} / \mathbf{P}^{2}}(d)\right)=0, \operatorname{deg}\left(X^{\prime} \cap L\right)=\operatorname{deg}(X \cap L)$. Finally we show that for $\alpha, \beta$ we can take the values $\alpha_{0}$ and $\beta_{0}$, respectively.

Step 1. In the case $X \in \mathcal{G S}_{1}$ the proof of Lemma 3.1 gives sufficient conditions on $\alpha, \beta$, namely (3.8), (3.9) in the Steps 2, 3, and the inequality (3.13) in Step 4. Due to (3.14), it is sufficient to check the inequality (3.13) after removing the term $d$ and substituting $d \sqrt{\beta} /(\sqrt{\beta}+1)$ for $\operatorname{mt} X$ and $m$, or, equivalently, to have

$$
\beta \leq\left(\frac{\sqrt{4 \alpha^{3}+\alpha^{2}-4 \alpha}+\alpha-2}{2\left(1+\alpha+\alpha^{2}\right)}\right)^{2} .
$$

Step 2. Assume that $X \in \mathcal{G S} \backslash \mathcal{G S}_{1}$. Since $\mathrm{mt}_{s} X \leq \mathrm{mt} X$, we can perform the inductive procedure described in the proof of Lemma 3.1 under assumptions (3.8), (3.9), (4.3), until the following situation occurs:

(1) $X$ satisfies (4.2);

(2) let $T_{X}^{*} \cap L=\left\{q_{1}, \ldots, q_{N}\right\}$, such that for each branch $D$ going through $q:=q_{N}$

$$
\operatorname{mt}\left(D_{\left(q_{1}\right)}, q_{1}\right)=\ldots=\operatorname{mt}\left(D_{\left(q_{N-1}\right)}, q_{N-1}\right)>\operatorname{mt}\left(D_{(q)}, q\right)>0
$$

(especially $D$ is not a smooth branch), and

$$
\operatorname{deg}(X \cap L)<d-\alpha \frac{\operatorname{deg} X}{d}-m_{q},
$$

where $m_{q}$ is the multiplicity of $X$ at $q$. 
Again, in the induction step, it is sufficient to show the two inequalities

$$
\begin{gathered}
\operatorname{deg} X-\operatorname{deg}(X \cap L) \leq \beta\left(d-1-\operatorname{mt}(X: L)-\operatorname{mt}_{s}(X: L)\right)^{2} \\
\operatorname{deg}((X: L) \cap L) \leq \operatorname{deg}(X \cap L) \leq d-1-\alpha \frac{\operatorname{deg}(X: L)}{d-1}
\end{gathered}
$$

Consider the possible situations.

Step 3. Under the hypotheses of the second step, assume that

$$
\operatorname{deg}(X \cap L) \geq d-2 m^{\prime}-\alpha \frac{\operatorname{deg} X}{d-m^{\prime}},
$$

where $m^{\prime}$ is the sum of the multiplicities of all branches, going through $q$.

Then the first inequality in 4.5 will follow from

$$
\alpha \frac{\beta\left(d-\mathrm{mt} X-\mathrm{mt}_{s} X\right)^{2}}{d-m^{\prime}} \leq\left(d-2 m^{\prime}\right)+\beta-2 \beta\left(d-\mathrm{mt} X-\mathrm{mt}_{s} X\right) .
$$

Since $m^{\prime} \leq \mathrm{mt}_{s} X \leq \mathrm{mt} X$, replacing the left-hand side by $\alpha \beta\left(d-\mathrm{mt} X-\mathrm{mt}_{s} X\right)$, and replacing the term $d-2 m^{\prime}$ in the right-hand side by $d-\mathrm{mt} X-\mathrm{mt}_{s} X$, one obtains a stronger inequality, namely

$$
0 \leq \beta+(1-2 \beta-\alpha \beta)\left(d-\mathrm{mt} X-\mathrm{mt}_{s} X\right),
$$

which is an immediate consequence of (3.8).

The second inequality of (4.5) will follow from

$$
d-1-\alpha \frac{\operatorname{deg} X-\operatorname{deg}(X \cap L)}{d-1} \geq \operatorname{deg}(X \cap L) .
$$

We replace $\operatorname{deg} X$ and $\operatorname{deg}(X \cap L)$ by the upper bounds (4.2), (4.4) and obtain

$$
(\alpha-1) d^{2}+d \geq \beta\left(\alpha+\alpha^{2}\right)\left(d-\operatorname{mt} X-\mathrm{mt}_{s} X\right)^{2}-m_{q}(d-1-\alpha),
$$

which holds true by (3.9).

Step 4. Under the hypotheses of the second step, assume that

$$
\operatorname{deg}(X \cap L)<d-2 m^{\prime}-\alpha \frac{\operatorname{deg} X}{d-m^{\prime}} .
$$

In this case we have to exert ourselves to obtain an analogue to the first inequality in (4.5). For that, we shall perform the following $m^{\prime}$-step algorithm.

Let $1 \leq j \leq m^{\prime}$ and let $X_{j-1} \in \mathcal{G S}$ be defined by a germ $\left(C_{j-1}, z\right)$ and a tree $T_{j-1}^{*}$ $\left(X_{0}=X, C_{0}=C, T_{0}^{*}=T^{*}\right)$, such that at the endpoint $q$ of $T_{j-1}^{*} \cap L$ the strict transform of $C_{j-1}$ has the multiplicity $m_{q}^{(j-1)}\left(m_{q}^{(0)}=m_{q}\right)$, and

$$
\operatorname{deg}\left(X_{j-1} \cap L\right)<d-m^{\prime}-\alpha \frac{\operatorname{deg} X}{d-m^{\prime}} .
$$

The $j$-th step of the algorithm appears as follows: introduce

$$
s_{j}:=\min \left\{l \geq 0 \mid \operatorname{deg}\left(X_{j-1} \cap L\right)+l m_{j}^{\prime} \geq d-2 m^{\prime}-\alpha \frac{\operatorname{deg} X}{d-m^{\prime}}\right\},
$$


where $m_{j}^{\prime}$ is the sum of the multiplicities of all branches of $\left(C_{j-1}, z\right)$ going through $q$ at the preceding point $\bar{q} \in T_{j-1}^{*} \cap L$. In particular, $m_{1}^{\prime}=m^{\prime}$ and $s_{1} \geq 1$. Define $X_{j-1}^{\prime}$ as the extension

$$
X_{j-1}^{\prime}:=X_{j-1} \underbrace{(q) \ldots(q)}_{s_{j} \text { times }}, \text { and } X_{j}:=X_{j-1}^{\prime}: L
$$

Note that in the previous formula, in the definition of $X_{j-1}$ and in the assumption of Step 2, we denote different points by $q$. But all these points appear in the extension operation introduced above, and the notation $q$ moves to new points of new schemes as was described in the assertion of Lemma 2.22.

Due to Lemma 2.24, again it is enough to show

$$
\begin{aligned}
\operatorname{deg}\left(X_{m^{\prime}} \cap L\right) & \leq d-m^{\prime}-\alpha \frac{\operatorname{deg} X_{m^{\prime}}}{d-m^{\prime}} \\
\operatorname{deg} X_{m^{\prime}} & \leq \beta\left(d-m^{\prime}-\operatorname{mt} X_{m^{\prime}}-\mathrm{mt}_{s} X_{m^{\prime}}\right)^{2}
\end{aligned}
$$

to complete the induction step.

We define the set

$$
\Lambda:=\left\{j \in\left[1, m^{\prime}-1\right] \mid m_{j+1}^{\prime}<m_{j}^{\prime}\right\}=\left\{j_{1}, j_{2}, \ldots, j_{\ell}\right\}
$$

$j_{k+1}>j_{k}$, that is, by Proposition 2.11 (ii), we have

$$
m_{j_{k}+1}^{\prime}=m_{j_{k}+2}^{\prime}=\ldots=m_{j_{k+1}}^{\prime}=m^{\prime}-k
$$

for any $k=0, \ldots, \ell\left(\right.$ where $j_{0}:=0$ and $\left.j_{\ell+1}:=m^{\prime}\right)$. We set

$$
N_{k}:=\sum_{i=j_{k}+1}^{j_{k+1}} s_{i}
$$

Note that if $j \in \Lambda$, that is, if $m_{j+1}^{\prime}=m_{j}^{\prime}-1$, then there are two possibilities: first, it might be that $\mathrm{mt} X_{j}=\mathrm{mt} X_{j-1}-1$. Secondly, if this is not the case, then Lemma 2.16 (ii) gives at least the existence of a branch $Q^{\prime}$ of the germ $\left(C_{j}, z\right)$ with $T_{j}^{*} \cap Q^{\prime} \subset T_{j}^{*} \cap L$, and Lemma 2.15 implies that $\mathrm{mt} X_{j+1}=\mathrm{mt} X_{j}-1$. In any case, we have $\operatorname{mt}_{s} X_{j}=\mathrm{mt}_{s} X_{j-1}-1$. Hence, we can estimate

$$
\tilde{\ell}:=\left(\operatorname{mt} X+\mathrm{mt}_{s} X\right)-\left(\operatorname{mt} X_{m^{\prime}}+\mathrm{mt}_{s} X_{m^{\prime}}\right) \begin{cases}\geq 0 & \text { if } \ell=0 \\ \geq \ell+1 & \text { if } \ell \neq 0\end{cases}
$$

To run an induction step, it is sufficient to show that

$$
\operatorname{deg} X_{m^{\prime}} \leq \operatorname{deg} X, \quad \operatorname{deg} X_{m^{\prime}} \leq \beta\left(d-m^{\prime}+\tilde{\ell}-m t X-\mathrm{mt}_{s} X\right)^{2}
$$


By construction, we have

$$
\begin{aligned}
\operatorname{deg} X_{m^{\prime}}= & \operatorname{deg} X+\sum_{k=0}^{\ell} N_{k} \frac{\left(m^{\prime}-k\right)\left(m^{\prime}-k+1\right)}{2}-\sum_{j=0}^{m^{\prime}-1} \operatorname{deg}\left(X_{j}^{\prime} \cap L\right) \\
= & \operatorname{deg} X+\frac{\left(m^{\prime}-\ell\right)\left(m^{\prime}-\ell+1\right)}{2} \cdot \sum_{k=0}^{\ell} N_{k}-\sum_{j=0}^{m^{\prime}-1} \operatorname{deg}\left(X_{j}^{\prime} \cap L\right) \\
& +\sum_{k=0}^{\ell-1}\left(\left(m^{\prime}-k\right) \cdot\left(N_{0}+\ldots+N_{k}\right)\right),
\end{aligned}
$$

and we can estimate

$$
\operatorname{deg}\left(X_{j_{k+1}-1} \cap L\right) \geq\left(m^{\prime}-k\right) \cdot\left(1+N_{0}+\ldots+N_{k}\right),
$$

for any $k=0, \ldots, \ell-1$. On the other hand, in the $j$-th step we have

$$
d-2 m^{\prime}+m_{j}^{\prime}-\alpha \frac{\operatorname{deg} X}{d-m^{\prime}}>\operatorname{deg}\left(X_{j-1}^{\prime} \cap L\right) \geq d-2 m^{\prime}-\alpha \frac{\operatorname{deg} X}{d-m^{\prime}},
$$

$j=0, \ldots, m^{\prime}-1$. In particular, this together with (4.10) implies that

$$
\left(m^{\prime}-\ell\right) \cdot \sum_{k=0}^{\ell} N_{k} \leq \operatorname{deg}\left(X_{m^{\prime}-1} \cap L\right)-\left(m^{\prime}-\ell\right)<d-2 m^{\prime}-\alpha \frac{\operatorname{deg} X}{d-m^{\prime}} .
$$

Hence, we can estimate

$$
\begin{aligned}
\operatorname{deg} X_{m^{\prime}} & \leq \operatorname{deg} X+\frac{m^{\prime}-\ell+1}{2} \cdot\left(m^{\prime}-\ell\right) \cdot \sum_{k=0}^{\ell} N_{k}-\sum_{\substack{k=0 \\
k+1 \notin \Lambda}}^{m^{\prime}-1} \operatorname{deg}\left(X_{k}^{\prime} \cap L\right) \\
& \leq \operatorname{deg} X+\left(\frac{m^{\prime}-\ell+1}{2}-m^{\prime}+\ell\right) \cdot\left(d-2 m^{\prime}-\alpha \frac{\operatorname{deg} X}{d-m^{\prime}}\right) \\
& \leq \operatorname{deg} X-\frac{m^{\prime}-1-\ell}{2} \cdot\left(d-2 m^{\prime}-\alpha \frac{\operatorname{deg} X}{d-m^{\prime}}\right),
\end{aligned}
$$

whence the first inequality in (4.9). The second will follow from

$$
\begin{aligned}
& \beta\left(d-m^{\prime}+\tilde{\ell}-\mathrm{mt} X-\mathrm{mt}_{s} X\right)^{2} \\
& \quad \geq \beta\left(d-\mathrm{mt} X-\mathrm{mt}_{s} X\right)^{2}-\frac{m^{\prime}-1-\ell}{2} \cdot\left(d-2 m^{\prime}-\alpha \frac{\operatorname{deg} X}{d-m^{\prime}}\right)
\end{aligned}
$$

or, equivalently,

$$
\begin{aligned}
& 2 \beta\left(m^{\prime}-\tilde{\ell}\right)\left(d-\operatorname{mt} X-\mathrm{mt}_{s} X\right) \\
& \leq \frac{m^{\prime}-1-\ell}{2} \cdot\left(d-2 m^{\prime}-\alpha \beta \frac{\left(d-\mathrm{mt} X-\mathrm{mt}_{s} X\right)^{2}}{d-m^{\prime}}\right)+\left(m^{\prime}-\tilde{\ell}\right)^{2} .
\end{aligned}
$$

Since $m^{\prime} \leq \mathrm{mt}_{s} X \leq \mathrm{mt} X$, this holds whenever

$$
0 \leq \frac{1-\alpha \beta}{4 \beta} \cdot\left(m^{\prime}-1-\ell\right)\left(d-2 m^{\prime}\right)-\left(m^{\prime}-\tilde{\ell}\right)\left(d-2 m^{\prime}\right) .
$$

Note that for fixed $\ell$ the right-hand side takes its minimum for the minimal possible value of $\tilde{\ell}$. Hence, it suffices to consider two cases: 
Case $4 A . \tilde{\ell}=\ell=0$. Then, since $m^{\prime} \geq 2,4.11$ is implied by

$$
\frac{1}{\beta} \geq 8+\alpha
$$

Case $4 B . \tilde{\ell}=\ell+1 \geq 2$. Then (4.11) holds if $\frac{1}{\beta} \geq 4+\alpha$, which is a consequence of (4.12).

Step 5. Finally we look for the maximal value of $\beta$, satisfying (3.8), (3.9), (4.3), (4.12) with any $\alpha>1$. It is easily shown that $\beta_{0}$ mentioned in the assertion of Lemma 4.1 is this maximal value.

Step 6. The induction base for $d \leq 6$ is trivial. Indeed, for $X \in \mathcal{G S} \backslash \mathcal{G S}$ the righthand side of $(4.2)$ does not exceed $\beta_{0}(6-2-2)^{2}<1$.

\section{Existence of Curves With one Singular Point}

We start with the following auxiliary statement:

Lemma 5.1. Let a scheme $Y \in \mathcal{G S} \cap \mathcal{S}$ be defined by a germ $(C, z)$ with branches $Q_{1}, \ldots, Q_{p}$ and the tree $T^{*}=T^{*}(C)$. Let $f \in J_{Y}$ satisfy

$$
\operatorname{mt}(f, z)=\operatorname{mt} Y, \quad\left(f, Q_{i}\right)>\sum_{q \in T^{*} \cap Q_{i}} m_{q} \cdot \operatorname{mt}\left(Q_{i,(q)}, q\right), i=1, \ldots, p .
$$

Then the germ $(f, z)$ also defines the singularity scheme $Y$, in particular $(f, z)$ and $(C, z)$ have the same topological type (which we denote by $Y$ ).

Proof. Step 1. In the case $Y \in \mathcal{G S}_{1} \cap \mathcal{S}$ this easily can be shown by induction on $\operatorname{deg} Y$. The induction base with $Y=\emptyset$ and $C$ being non-singular at $z$, is trivial. Hence, assume $\operatorname{deg} Y>0$ and blow up the point $z$. If $w$ is an intersection point of the strict transform $C^{*}$ of $C$ with the exceptional divisor $E$, then, for any branch $Q_{i,(w)}$ of $C^{*}$ centred at $w$, we have

$$
\left(f_{(w)}, Q_{i,(w)}\right)=\left(f \cdot Q_{i}\right)-\operatorname{mt}(f, z)>\sum_{q \in T^{*} \cap Q_{i} \backslash\{z\}} m_{q} \geq 0 .
$$

Thus, $w \in f_{(w)}$ and, if $C^{*}$ is non-singular at $w$, then $\operatorname{mt}\left(f_{(w)}, w\right) \geq 1=\operatorname{mt}\left(C^{*}, w\right)$. If $C^{*}$ is singular at $w$, then by Lemma 2.8, $f_{(w)} \in J_{Y_{(w)}^{*}}$, where $Y_{(w)}^{*} \in \mathcal{G S}_{1}$ is defined by the germ $\left(C^{*}, w\right)$ and its tree of essential points. Especially, again, $\operatorname{mt}\left(f_{(w)}, w\right) \geq \operatorname{mt}\left(C^{*}, w\right)$. On the other hand,

$$
\operatorname{mt}(f, z) \geq \sum_{w \in C^{*} \cap E} \operatorname{mt}\left(f_{(w)}, w\right) \geq \sum_{w \in C^{*} \cap E} \operatorname{mt}\left(C^{*}, w\right)=\left(C^{*}, E\right)=\operatorname{mt} Y,
$$

which implies $\operatorname{mt}\left(f_{(w)}, w\right)=\operatorname{mt}\left(C^{*}, w\right)$ and, together with 5.3 and the induction assumption, $\left(f_{(w)}, w\right)$ defines the same singularity scheme as $\left(C^{*}, w\right)$. Since, moreover, $C^{*}$ and $f_{(w)}$ are transversal to $E$ at $w,(f, z)$ and $(C, z)$ define the same singularity scheme $Y$. 
Remark. Let $(C, z)$ be given in local coordinates $x, y$ at $z$ by

$$
C(x, y)=\prod_{i=1}^{m}\left(y-\sum_{j=1}^{\infty} a_{i j} x^{j}\right)
$$

and define the essential part $\Gamma_{e s}$ of the Newton diagram $\Gamma$ of $C(x, y)$ at the origin as the union of

(i) all the integral points $(i, j) \in \Gamma$ with positive $i, j$,

(ii) a point $(n, 0) \in \Gamma$, if it is not an endpoint of an edge $\left[(n, 0),\left(n^{\prime}, 1\right)\right] \subset \Gamma$,

(iii) a point $(0, n) \in \Gamma$, if it is not an endpoint of an edge $\left[(0, n),\left(1, n^{\prime}\right)\right] \subset \Gamma$.

We claim that $f(x, y)$ has the same essential part $\Gamma_{e s}$ of the Newton diagram at the origin. This is easily shown by induction on $\operatorname{deg} Y$, using the transformation

$$
(x, y) \mapsto(x, x y)
$$

as the blowing-up at $z$.

Step 2. Now assume that $Y$ is arbitrary in $\mathcal{G S} \cap \mathcal{S}$. We apply induction on the number

$$
N:=\sum_{Q} \sum_{q \in T^{*} \cap Q} \operatorname{mt}\left(Q_{(q)}, q\right),
$$

where $Q$ runs through all singular branches of $C$. The case $N=0$ means just $Y \in \mathcal{G S}_{1}$. If $N>0$ then, again, we blow up the point $z$. Each intersection point of the strict transform $C^{*}$ of $C$ with the exceptional divisor $E$ corresponds to a straight line $W$ through $z$, tangent to $C$. Without restriction, we can suppose that in local coordinates $x, y$ at $z$ we have $W=y$ and $C(x, y)$ decomposes in local branches $Q_{1}, \ldots, Q_{p}$ with

$$
Q_{k}=\prod_{i=1}^{s_{k}}\left(y-\xi_{i}^{(k)}(x)\right), \quad \xi_{i}^{(k)}(x)=\sum_{j=1}^{\infty} a_{i j}^{(k)} x^{j / s_{k}}, \quad s_{k}=\operatorname{mt}\left(Q_{k}, z\right)
$$

The (covering) transformation

$$
(x, y) \mapsto\left(x^{M}, y\right) \text { with } M:=\prod_{k=1}^{p} s_{k}
$$

turns $(C, z)$ into a germ $(\widetilde{C}, z)$ with multiplicity $\operatorname{mt}(\widetilde{C}, z)=\operatorname{mt}(C, z)=\operatorname{mt} Y$ and only non-singular branches

$$
Q_{i}^{(k)}=y-\sum_{j=1}^{\infty} a_{i j}^{(k)} x^{j M / s_{k}}, \quad i=1, \ldots, s_{k}, k=1, \ldots, p .
$$

Let $\widetilde{Y} \in \mathcal{G} \mathcal{S}_{1}$ be defined by the germ $(\widetilde{C}, z)$ and the tree $T^{*}(\widetilde{C})$. We shall show that the transform $\widetilde{\varphi}(x, y):=\varphi\left(x^{M}, y\right)$ of any element $\varphi \in J_{Y}$ belongs to $J_{\widetilde{Y}}$.

By Lemma 2.8, we have for any (fixed) $i=1, \ldots, s_{k}$,

$$
\left(\widetilde{\varphi}, Q_{i}^{(k)}\right)=\frac{M}{s_{k}}\left(\varphi, Q_{k}\right) \geq \frac{M}{s_{k}}\left(2 \delta\left(Q_{k}\right)+\sum_{l \neq k}\left(Q_{l}, Q_{k}\right)+\sum_{q \in T * \cap Q_{k}} \operatorname{mt}\left(Q_{k,(q)}, q\right)\right) .
$$


Hence, using the considerations in the proof of Lemma 2.19,

$$
\begin{aligned}
\left(\widetilde{\varphi}, Q_{i}^{(k)}\right) \geq & \left(\sum_{j \neq i}\left(Q_{i}^{(k)}, Q_{j}^{(k)}\right)-M+\frac{M}{s_{i}}\right)+\left(\sum_{l \neq k} \sum_{j=1}^{s_{l}}\left(Q_{i}^{(k)}, Q_{j}^{(l)}\right)\right) \\
& +\left(\max _{(j, l) \neq(i, k)}\left(Q_{i}^{(k)}, Q_{j}^{(l)}\right)+M-\frac{M}{s_{i}}\right) \\
= & \sum_{(j, l) \neq(i, k)}\left(Q_{i}^{(k)}, Q_{j}^{(l)}\right)+\#\left\{q \in T^{*}(\widetilde{C}) \cap Q_{i}^{(k)}\right\},
\end{aligned}
$$

and Lemma 2.8 implies $\widetilde{\varphi} \in J_{\widetilde{Y}}$.

The germ $\tilde{f}(x, y)=f\left(x^{M}, y\right)$, clearly, satisfies $\operatorname{mt}(\tilde{f}, z)=\operatorname{mt} Y$. Due to (5.2), the previous computation with $\widetilde{f}$ instead of $\widetilde{\varphi}$ gives

$$
\left(\tilde{f}, Q_{i}^{(k)}\right)>\sum_{q \in T^{*}(\widetilde{C}) \cap Q_{i}^{(k)}} \operatorname{mt}\left(\widetilde{C}_{(q)}, q\right), \quad i=1, \ldots, s_{k}, k=1, \ldots, p .
$$

Hence, by Step $1,(\widetilde{f}, z)$ defines the same singularity scheme as $(\widetilde{C}, z)$. Denote by $\Gamma$ the Newton diagram of $C(x, y)$ at the origin. Evidently, the Newton diagram $\widetilde{\Gamma}$ of $\widetilde{C}$ and its essential part $\widetilde{\Gamma}_{e s}$ are obtained from $\Gamma, \Gamma_{e s}$ by the transformation $(I, J) \mapsto(M I, J)$. As established above, $\tilde{f}$ has the same essential part $\widetilde{\Gamma}_{e s}$ of the Newton diagram at the origin. Therefore, $\Gamma_{e s}$ is the essential part of the Newton diagram of $f(x, y)$ at the origin.

Let $\Gamma^{\prime}$ be the part of $\Gamma$ corresponding to the branches of $C$ tangent to $y$, and

$$
\left(n_{1}, m_{1}\right), \ldots,\left(n_{l}, m_{l}\right), \quad m_{1}>\cdots>m_{l}=0,
$$

be the vertices of $\Gamma^{\prime}$. Applying the blowing-up (5.4) at $z$, we easily obtain that the Newton diagram of $C^{*}(x, y)$ at $w=(0,0)$ has the vertices

$$
\left(0, m_{1}\right),\left(n_{2}-n_{1}, m_{2}\right), \ldots,\left(n_{l-1}-n_{1}, m_{l-1}\right),\left(n_{l}-n_{1}, 0\right),
$$

and that $f_{(w)}(x, y)$ has the Newton diagram with vertices

$$
\left(0, m_{1}\right),\left(n_{2}-n_{1}, m_{2}\right), \ldots,\left(n_{l-1}-n_{1}, m_{l-1}\right),(r, 0),
$$

where $r$ may be different from $n_{l}-n_{1}$ only in the case $m_{l-1}=1$. In particular, this means that

$$
\operatorname{mt}\left(f_{(w)}, w\right)=\operatorname{mt}\left(C^{*}, w\right), \quad\left(f_{(w)}, E\right)=\operatorname{mt}\left(C^{*} \cap E, w\right) .
$$

On the other hand, for any branch $Q_{i,(w)}$ of $C^{*}$ centred at $w$, we have

$$
\left(f_{(w)}, Q_{i,(w)}\right)=\left(f, Q_{i}\right)-\operatorname{mt}(f, z) \cdot \operatorname{mt}\left(Q_{i}, z\right)>\sum_{q \in T^{*} \cap Q_{i} \backslash\{z\}} m_{q} \cdot \operatorname{mt}\left(Q_{i,(q)}, q\right) .
$$

This, together with (5.6) and the induction assumption, implies that $\left(f_{(w)} \cdot E, w\right)$ defines the same singularity scheme as $\left(C^{*} \cdot E, w\right)$.

Finally, blowing down all the germs $f_{(w)}, w \in C^{*} \cap E$, one obtains that $(f, z)$ also defines the singularity scheme $Y$. 
Definition 5.7. Let $F$ be a curve of degree $d$ with an isolated singular point $z$. The germ $\left(H_{\mathbf{P}^{2}}^{e s, d}, F\right)$ of the equisingular stratum $H_{\mathbf{P}^{2}}^{e s, d} \subset \mathbf{P}^{N}=\mathbf{P}\left(\Gamma\left(\mathcal{O}_{\mathbf{P}^{2}}(d)\right)\right)$, $N=d(d+3) / 2$, at $F$ (cf. $\mathbf{G r L})$ is called $\mathbf{T}-\mathbf{s m o o t h}$, if it is smooth and, for any $d^{\prime}>d$, it is a transversal intersection in $\mathbf{P}\left(\Gamma\left(\mathcal{O}_{\mathbf{P}^{2}}\left(d^{\prime}\right)\right)\right)$ of $\left(H_{\mathbf{P}^{2}}^{e s, d^{\prime}}, F\right)$ and $\mathbf{P}\left(\Gamma\left(\mathcal{O}_{\mathbf{P}^{2}}(d)\right)\right)$, included in $\mathbf{P}\left(\Gamma\left(\mathcal{O}_{\mathbf{P}^{2}}\left(d^{\prime}\right)\right)\right)$ by $C \mapsto C L^{d^{\prime}-d}$, where $L$ is a fixed generic straight line not passing through $z$.

Lemma 5.8. For any scheme $X \in \mathcal{G S} \cap \mathcal{S}$, and any positive integer $d$, satisfying

$$
\operatorname{deg} X+\operatorname{mt} X+1< \begin{cases}(3-2 \sqrt{2})(d-\mathrm{mt} X-2)^{2}, & \text { if } X \in \mathcal{G} \mathcal{S}_{1}, \\ \beta_{0}\left(d-\mathrm{mt} X-\mathrm{mt}_{s} X-2\right)^{2}, & \text { if } X \in \mathcal{G S} \backslash \mathcal{G} \mathcal{S}_{1},\end{cases}
$$

$\beta_{0}$ as above in Lemma 4.1, there exists an irreducible curve $F$ of degree $d$ with a singular point $z$ of (topological) type $X$ as its only singularity such that the germ $\left(H_{\mathbf{P}^{2}}^{e s,}, F\right)$ is T-smooth.

Proof. Step 1. Let $(C, z)$ be a defining germ of the scheme $X$ and consider the germ $(\widetilde{C}, z), \widetilde{C}=C \cdot L$, where $L$ is a generic straight line through $z$. Note that

$$
T^{*}(\widetilde{C})=T^{*}(C), \quad \operatorname{mt}(\widetilde{C}, z)=\operatorname{mt}(C, z)+1 .
$$

Now we introduce (1) the scheme $X^{\prime}$, defined by the germ $(C, z)$ and the tree $T_{X^{\prime}}^{*}$, containing $T^{*}(C)$ and the first non-essential points of all local branches of $(C, z)$, and (2) the scheme $\widetilde{X}$, defined by the germ $(\widetilde{C}, z)$ and the tree $T^{*}(C)$. Clearly,

$$
\begin{aligned}
\operatorname{mt} X^{\prime}=\operatorname{mt} X, & \operatorname{mt} \tilde{X}=\operatorname{mt} X+1, \\
\operatorname{deg} X^{\prime} \leq \operatorname{deg} X+\operatorname{mt} X, & \operatorname{deg} \tilde{X} \leq \operatorname{deg} X+\operatorname{mt} X+1,
\end{aligned}
$$

and in addition, for any local branch $Q$ of $(C, z)$ and any elements $f \in \mathcal{J}_{X^{\prime} / \mathbf{P}^{2}, z}$, $g \in \mathcal{J}_{\widetilde{X} / \mathbf{P}^{2}, z}$,

$$
\begin{gathered}
(f, Q)>\sum_{q \in T^{*}(C) \cap Q} \operatorname{mt}\left(C_{(q)}, q\right), \\
(g, Q) \geq \sum_{q \in T^{*}(C) \cap Q} \operatorname{mt}\left(\widetilde{C}_{(q)}, q\right)>\sum_{q \in T^{*}(C) \cap Q} \operatorname{mt}\left(C_{(q)}, q\right) .
\end{gathered}
$$

By (5.9), (5.10) and the Lemmas 3.1, 4.1, we may assume that

$$
h^{1}\left(\mathcal{J}_{X^{\prime} / \mathbf{P}^{2}}(d-1)\right)=h^{1}\left(\mathcal{J}_{\widetilde{X} / \mathbf{P}^{2}}(d-1)\right)=0 .
$$

Hence, due to $X^{\prime} \subsetneq \widetilde{X}$, there exists a (generic) curve

$$
f \in H^{0}\left(\mathcal{J}_{X^{\prime} / \mathbf{P}^{2}}(d-1)\right) \backslash H^{0}\left(\mathcal{J}_{\tilde{X} / \mathbf{P}^{2}}(d-1)\right),
$$

which, by (5.10), (5.11), satisfies the condition of Lemma 5.1. Thus, $(f, z)$ defines the singularity scheme $X$. Replacing, if necessary, multiple components of $f$ (which do not go through $z$ ) by distinct components, we obtain a reduced curve $f$ of degree $d-1$.

If $f$ is irreducible, then we are done. Otherwise, we shall use Bertini's Theorem to construct the desired irreducible curve $F$ of degree $d$. For that, let a straight line $L$ meet $f$ at $d-1$ distinct non-singular points $w_{1}, \ldots, w_{d-1}$. Obviously, 
$h^{1}\left(\mathcal{J}_{\left\{w_{i}\right\} / L}(1)\right)=0$ for each $i=1, \ldots, d-1$ and, by 5.12) $h^{1}\left(\mathcal{J}_{X^{\prime} / f}(d-1)\right)=0$. First, observe that this implies

$$
h^{1}\left(\mathcal{J}_{X^{\prime} \cup\left\{w_{i}\right\} / f L}(d)\right)=0, \quad 1 \leq i \leq d-1 .
$$

Indeed, the first morphism $\operatorname{id}_{1} \otimes L+f \otimes \mathrm{id}_{2}$ in the exact sequence

$$
0 \longrightarrow \mathcal{O}_{f}(d-1) \oplus \mathcal{O}_{L}(1) \longrightarrow \mathcal{O}_{f L}(d) \longrightarrow \mathcal{O}_{f \cap L} \longrightarrow 0
$$

maps the sheaf $\mathcal{J}_{X^{\prime} / f}(d-1) \oplus \mathcal{J}_{\left\{w_{i}\right\} / L}(1)$ injectively to the sheaf $\mathcal{J}_{X^{\prime} \cup\left\{w_{i}\right\} / f L}(d)$. Now, consider the commutative diagram

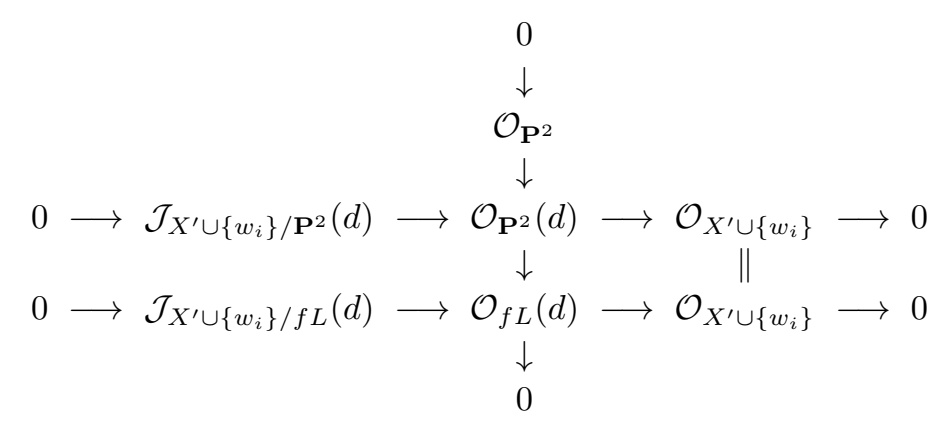

to deduce from (5.14) the surjectivity of $H^{0}\left(\mathcal{O}_{\mathbf{P}^{2}}(d)\right) \rightarrow H^{0}\left(\mathcal{O}_{X^{\prime} \cup\left\{w_{i}\right\}}\right)$, which is equivalent to

$$
h^{1}\left(\mathcal{J}_{X^{\prime} \cup\left\{w_{i}\right\} / \mathbf{P}^{2}}(d)\right)=0, \quad 1 \leq i \leq d-1 .
$$

In particular, there exist curves

$$
\varphi_{i} \in H^{0}\left(\mathcal{J}_{X^{\prime} / \mathbf{P}^{2}}(d)\right) \backslash H^{0}\left(\mathcal{J}_{X^{\prime} \cup\left\{w_{i}\right\} / \mathbf{P}^{2}}(d)\right), \quad 1 \leq i \leq d-1,
$$

and, by Bertini's theorem, the generic member $\Phi$ of the linear family

$$
f \cdot L+\lambda_{1} \varphi_{1}+\cdots+\lambda_{d-1} \varphi_{d-1}
$$

is irreducible and smooth outside of the base points, whereas at $z$ it has a singularity of the (topological) type $X$. Assume that $\Phi$ has singular points $w_{1}, \ldots, w_{p}$ different from $z$. Using the preceding arguments, we show that there exist curves

$$
\Phi_{j} \in H^{0}\left(\mathcal{J}_{X^{\prime} / \mathbf{P}^{2}}(d)\right) \backslash H^{0}\left(\mathcal{J}_{X^{\prime} \cup\left\{w_{j}\right\} / \mathbf{P}^{2}}(d)\right), \quad j=1, \ldots, p .
$$

Finally, by Bertini's theorem, a generic member $F$ of the linear family

$$
f \cdot L+\lambda_{1} \varphi_{1}+\cdots+\lambda_{d-1} \varphi_{d-1}+\mu_{1} \Phi_{1}+\cdots+\mu_{p} \Phi_{p}
$$

has no singularities outside $z$.

Step 2. Note that, by Lemma 2.7, $h^{1}\left(\mathcal{J}_{X^{\prime} / \mathbf{P}^{2}}(d)\right)=0$ implies $h^{1}\left(\mathcal{J}^{\text {es }}(d)\right)=0$, where $\mathcal{J}^{\text {es }} \subset \mathcal{O}_{\mathbf{P}^{2}}$ is the ideal sheaf of the zero-dimensional scheme given by the equisingularity ideal $I^{\text {es }} \subset \mathcal{O}_{\mathbf{P}^{2}, z}$ of $(F, z)$. But the latter equality yields the required $\mathrm{T}$-smoothness of the germ $\left(H_{\mathbf{P}^{2}}^{e s, d}, F\right)$ (GrK, GrZ $)$. 


\section{Proof of Theorems 1 And 2}

Definition 6.1. Let $X \in \mathcal{S}$ be a singularity scheme. By $s(X)$ we denote the minimal integer such that there exists an irreducible curve $F$ of degree $s(X)$ with a singular point $z$ of type $X$ as its only singularity, and such that the germ $\left(H_{\mathbf{P}^{2}}^{e s, d}, F\right)$ of the equisingular stratum $H_{\mathbf{P}^{2}}^{e s, d} \subset \mathbf{P}^{N}=\mathbf{P}\left(\Gamma\left(\mathcal{O}_{\mathbf{P}^{2}}(d)\right)\right), N=d(d+3) / 2$, at $F$ is T-smooth.

Lemma 6.2. Let $X \in \mathcal{S}$ be a singularity scheme. Then $s(X) \leq \sigma(X)$, where

$$
\sigma(X):=\left\{\begin{array}{cl}
{[\sqrt{2} \mathrm{mt} X]+1,} & \text { if } X \text { is ordinary, } \\
{[(1+\sqrt{2}) \sqrt{\operatorname{deg} X+\mathrm{mt} X+1}]+\mathrm{mt} X+3,} & \text { if } X \in \mathcal{G S}_{1}, \\
{\left[\sqrt{(\operatorname{deg} X+\mathrm{mt} X+1) \beta_{0}^{-1}}\right]+\mathrm{mt} X+\mathrm{mt}_{s} X+3,} & \text { if } X \notin \mathcal{G} \mathcal{S}_{1} .
\end{array}\right.
$$

Proof. By Lemma 5.8, $s(X) \leq \sigma(X)$ for any non-ordinary singularity $X$. The case of an ordinary singularity was already treated in GLS and the result follows from Lemma 6.4 below.

Definition 6.3. Let $\bar{z}=\left(z_{1}, \ldots, z_{n}\right)$ be a tuple of distinct points in $\mathbf{P}^{2}$, and $\bar{m}=\left(m_{1}, \ldots, m_{n}\right)$ be a vector of positive integers, then we denote by $X(\bar{z}, \bar{m})$ the zero-dimensional scheme in $\mathbf{P}^{2}$ defined by the ideals $\mathfrak{m}_{z_{i}}^{m_{i}} \subset \mathcal{O}_{\mathbf{P}^{2}, z_{i}}, i=1, \ldots, n$.

Lemma 6.4 (GLS, section 3.3). Let $z_{1}, \ldots, z_{n}$ be distinct generic points in $\mathbf{P}^{2}$, and the positive integers $d, m_{1}, \ldots, m_{n}$ satisfy

$$
\sum_{i=1}^{n} \frac{m_{i}\left(m_{i}+1\right)}{2}<\frac{d^{2}+6 d-1}{4}-\left[\frac{d}{2}\right] .
$$

Then (1) there exists an irreducible curve $F_{d}$ of degree $d$ with ordinary singular points $z_{1}, \ldots, z_{n}$ of multiplicities $m_{1}, \ldots, m_{n}$, respectively, as its only singularities; (2) for $\bar{z}=\left(z_{1}, \ldots, z_{n}\right), \bar{m}=\left(m_{1}, \ldots, m_{n}\right)$,

$$
h^{1}\left(\mathcal{J}_{\left.X(\bar{z}, \bar{m}) / \mathbf{P}^{2}(d)\right)=0 .} .\right.
$$

Lemma 6.6. If a positive integer $d$ and the singularities $S_{1}, \ldots, S_{n}$ satisfy the inequality

$$
\sum_{i=1}^{n} \frac{\left(s\left(S_{i}\right)+1\right)\left(s\left(S_{i}\right)+2\right)}{2}<\frac{d^{2}+6 d-1}{4}-\left[\frac{d}{2}\right],
$$

then there exists an irreducible curve $F$ of degree $d$ with exactly $n$ singular points of (topological) types $S_{1}, \ldots, S_{n}$, respectively.

Proof. Due to Lefschetz's principle (cf. [JeD, Theorem 1.13), we suppose, without loss of generality, $\mathbf{K}=\mathbf{C}$. Let

$$
G_{i}(x, y)=\sum_{0 \leq k+l \leq s\left(S_{i}\right)} a_{k l}^{(i)} x^{k} y^{l}, \quad \operatorname{deg} G_{i}=s\left(S_{i}\right), \quad i=1, \ldots, n,
$$


be affine irreducible curves such that each of them has exactly one singular point at $(0,0)$ of type $S_{1}, \ldots, S_{n}$, respectively.

On the other hand, let $z_{1}, \ldots, z_{n}$ be distinct generic points in $\mathbf{P}^{2}$, then Lemma 6.4 implies the existence of an irreducible curve $G^{\prime}$ of degree $d$ having ordinary singularities at $z_{1}, \ldots, z_{n}$ of multiplicities $m_{i}=s\left(S_{i}\right)+1, i=1, \ldots, n$, as its only singularities. For any $i=1, \ldots, n$, let us fix affine coordinates $x_{i}, y_{i}$ in a neighbourhood of $z_{i}$. The relation (6.5) means that an affine neighbourhood $U$ of $G^{\prime}$ in $\mathbf{P}^{N}=\mathbf{P}\left(\Gamma\left(\mathcal{O}_{\mathbf{P}^{2}}(d)\right)\right)$ can be parametrized by the following independent parameters: to any $\Phi \in U$ we assign

(1) coefficients $A_{k l}^{(i)}, 0 \leq k+l \leq s\left(S_{i}\right)$, from the representation

$$
\Phi\left(x_{i}, y_{i}\right)=\sum_{0 \leq k+l \leq d} A_{k l}^{(i)} x_{i}^{k} y_{i}^{l}
$$

of $\Phi$ in the coordinates $x_{i}, y_{i}$, for any $i=1, \ldots, n$,

(2) some parameters $B_{j}, 1 \leq j \leq r:=N-\sum_{i=1}^{n}\left(s\left(S_{i}\right)+1\right)\left(s\left(S_{i}\right)+2\right) / 2$.

First we deform $G^{\prime}$ into a curve $G$ by addition of the leading form, that is, the part of degree $s\left(S_{i}\right)$, of $G_{i}(x, y)$ as the $s\left(S_{i}\right)$-jet at $z_{i}, i=1, \ldots, n$. Without loss of generality we may suppose that $G$ is irreducible and has no singularities outside $\left\{z_{1}, \ldots, z_{n}\right\}$. Moreover, in the local coordinates $x_{i}, y_{i}, G$ is represented as

$$
G^{(i)}\left(x_{i}, y_{i}\right)=\sum_{d \geq k+l \geq s\left(S_{i}\right)} a_{k l}^{(i)} x_{i}^{k} y_{i}^{l}
$$

$i=1, \ldots, n$, and corresponds to the parameter values $A_{k l}^{(i)}=0$, if $k+l<s\left(S_{i}\right)$, $A_{k l}^{(i)}=a_{k l}^{(i)}$, if $k+l=s\left(S_{i}\right)$, and $B_{j}=b_{j}, j=1, \ldots, r$. We shall look for the desired curve $F$ close to $G$, given by parameters

$$
A_{k l}^{(i)}(\tau)=\left\{\begin{array}{cc}
\tau^{s\left(S_{i}\right)-k-l} a_{k l}^{(i)}(\tau) & \text { if } k+l<s\left(S_{i}\right) \\
a_{k l}^{(i)}(\tau) & \text { if } k+l=s\left(S_{i}\right)
\end{array} \quad \text { and } B_{j}=b_{j}(\tau),\right.
$$

where $a_{k l}^{(i)}(\tau), b_{j}(\tau)$ are smooth functions in a neighbourhood of zero such that

$$
a_{k l}^{(i)}(0)=a_{k l}^{(i)}, \quad b_{j}(0)=b_{j},
$$

for all $i, j, k, l$.

Let us fix some $1 \leq i \leq n$. In a neighbourhood of the point $z_{i}$ we have

$$
F\left(x_{i}, y_{i}\right)=\sum_{0 \leq k+l \leq s\left(S_{i}\right)} \tau^{s\left(S_{i}\right)-k-l} a_{k l}^{(i)}(\tau) x_{i}^{k} y_{i}^{l}+\sum_{d \geq p+q>s\left(S_{i}\right)} a_{p q}^{(i)}(\tau) x_{i}^{p} y_{i}^{q},
$$

where the coefficients $a_{p q}^{(i)}(\tau), p+q>s\left(S_{i}\right)$, are affine functions in the parameters $A_{k l}^{(s)}, s \neq i$, and $B_{j}, j=1, \ldots, r$. The transformation $\left(x_{i}, y_{i}\right) \mapsto\left(\tau x_{i}, \tau y_{i}\right)$ turns $F$ for sufficiently small $\tau \neq 0$ into a curve

$$
F_{i}\left(x_{i}, y_{i}\right)=\sum_{0 \leq k+l \leq s\left(S_{i}\right)} a_{k l}^{(i)}(\tau) x_{i}^{k} y_{i}^{l}+\sum_{d \geq p+q>s\left(S_{i}\right)} \tau^{p+q-s\left(S_{i}\right)} a_{p q}^{(i)}(\tau) x_{i}^{p} y_{i}^{q}
$$


close to $G_{i}$ in $\mathbf{P}^{N}=\mathbf{P}\left(\Gamma\left(\mathcal{O}_{\mathbf{P}^{2}}(d)\right)\right)$. By the definition of $s\left(S_{i}\right)$, the germ of the equisingular stratum $H_{\mathbf{P}^{2}}^{e s, d} \subset \mathbf{P}^{N}$ at $G_{i}$ can be described by $c\left(S_{i}\right)$ equations

$$
\varphi_{u}^{(i)}\left(A_{k l}^{(i)}\right)=0, \quad u=1, \ldots, c\left(S_{i}\right)
$$

on the coefficients $A_{k l}^{(i)}, 0 \leq k+l \leq d$, of a curve

$$
H\left(x_{i}, y_{i}\right)=\sum_{0 \leq k+l \leq d} A_{k l}^{(i)} x_{i}^{k} y_{i}^{l}
$$

such that there exists $\Lambda_{i} \subset\left\{(k, l) \in \mathbf{Z}^{2} \mid k, l \geq 0, k+l \leq s\left(S_{i}\right)\right\}, \operatorname{card}\left(\Lambda_{i}\right)=c\left(S_{i}\right)$, with

$$
\operatorname{det}\left(\frac{\partial \varphi_{u}^{(i)}}{\partial A_{k l}^{(i)}}\right) \underbrace{}_{\substack{u=1, \ldots, c\left(S_{i}\right) \\(k, l) \in \Lambda_{i}}} \neq 0
$$

at the point $A_{k l}^{(i)}=a_{k l}^{(i)}, 0 \leq k+l \leq s\left(S_{i}\right)$ and $A_{p q}^{(i)}=0, p+q>s\left(S_{i}\right)$. Thus, the condition on $F$ to have singular points of types $S_{1}, \ldots, S_{n}$ can be expressed as the system of equations

$$
\varphi_{u}^{(i)}\left(\left\{a_{k l}^{(i)}(\tau) \mid k+l \leq s\left(S_{i}\right)\right\},\left\{\tau^{p+q-s\left(S_{i}\right)} a_{p q}^{(i)}(\tau) \mid p+q>s\left(S_{i}\right)\right\}\right)=0,
$$

$u=1, \ldots, c\left(S_{i}\right), i=1, \ldots, n$. From the above it follows immediately that at the point $\tau=0$ the determinant

$$
\operatorname{det}\left(\frac{\partial \varphi_{u}^{(i)}}{\partial a_{k l}^{(m)}}\right) \underbrace{}_{\substack{u=1, \ldots, c\left(S_{i}\right) \\(k, l) \in \Lambda_{m} \\ m, i=1, \ldots, n}}=\prod_{i=1}^{n} \operatorname{det}\left(\frac{\partial \varphi_{u}^{(i)}}{\partial a_{k l}^{(i)}}\right)_{\substack{u=1, \ldots, c\left(S_{i}\right) \\(k, l) \in \Lambda_{i}}}
$$

does not vanish, which implies the existence of an appropriate solution to (6.7), and, hereby, the existence of a curve $F$ with $n$ singular points of types $S_{1}, \ldots, S_{n}$.

The only thing we should explain, is why $F$ has no other singular points. Let us consider $F$ as a polynomial function. First, note that $F$ is a small deformation of the function $G$, which has $(d-1)^{2}-\left(s\left(S_{1}\right)-1\right)^{2}-\cdots-\left(s\left(S_{n}\right)-1\right)^{2}$ critical points out of the zero level. Second, we deform each ordinary critical point $z_{i}$ of $G$ by means of the function $G_{i}$ which has $\left(s\left(S_{i}\right)-1\right)^{2}-\mu\left(S_{i}\right)$ critical points out of the zero level. Hence, $F$ has at least $(d-1)^{2}-\mu\left(S_{1}\right)-\ldots-\mu\left(S_{n}\right)$ critical points out of the zero level, that means the $n$ constructed singular points are the only singular points of the curve $F$.

Thus, to prove the Theorems 1 and 2 from the introduction, by Lemma 6.2, it suffices to prove the following

Proposition 6.8. For any singularity $X \in \mathcal{S}$,

$$
(\sigma(X)+1)(\sigma(X)+2) \leq 196 \mu(X) .
$$

Proof. If $X$ is an ordinary singularity, then $\mu(X)=(\mathrm{mt} X-1)^{2}$; therefore

$$
(\sigma(X)+1)(\sigma(X)+2) \leq 2(\operatorname{mt} X+1)(\operatorname{mt} X+3) \leq 30 \mu(X) .
$$


For an arbitrary singularity $X \in \mathcal{S}$, defined by the germ $(C, z)$, we have

$$
\mu(X)=2 \delta(X)-r+1=\sum_{q \in T^{*}(C)} m_{q}\left(m_{q}-1\right)-r+1,
$$

where $r$ is the number of local branches. Note that the number of the essential points $q$ with $m_{q}=1$ does not exceed $\mathrm{mt} X<\sqrt{\mu(X)}+1$. Hence, by Lemma 2.6,

$$
2(\operatorname{deg} X+\operatorname{mt} X+1) \leq \sum_{m_{q}>1} m_{q}\left(m_{q}+1\right)+4 \operatorname{mt} X+2<3(\sqrt{\mu(X)}+\sqrt{2})^{2}
$$

thus

$$
\sigma(X)<\sqrt{\frac{\operatorname{deg} X+\mathrm{mt} X+1}{\beta_{0}}}+2 \mathrm{mt} X+3<\left(\sqrt{\frac{3}{2 \beta_{0}}}+2\right) \sqrt{\mu(X)}+\sqrt{\frac{3}{\beta_{0}}}+5,
$$

and, finally, it follows that $(\sigma(X)+1)(\sigma(X)+2)$ is smaller than

$\left(\sqrt{\frac{3}{2 \beta_{0}}}+2\right)^{2} \mu(X)+\left(\sqrt{\frac{3}{\beta_{0}}}+6\right)\left(\sqrt{\frac{3}{\beta_{0}}}+7\right)+\left(2 \sqrt{\frac{3}{\beta_{0}}}+13\right)\left(\sqrt{\frac{3}{2 \beta_{0}}}+2\right) \sqrt{\mu(X)}$,

which for $\mu(X) \geq 2$ does not exceed $196 \mu(X)$.

\section{REFERENCES}

[BrK] Brieskorn, E.; Knörrer, H.: Plane algebraic curves. Birkhäuser-Verlag (1986).

[Cas] Casas-Alvero, E.: Infinitely near imposed singularities and singularities of polar curves. Math. Ann. 287, 429-454 (1990).

[DiH] Diaz, S.; Harris, J.: Ideals associated to deformations of singular plane curves. Trans. Amer. Math. Soc., 309, No. 2, 433-467 (1988).

[GrK] Greuel, G.-M.; Karras, U.: Families of varieties with prescribed singularities. Compos. math. 69 no. 1, 83-110 (1989).

[GrL] Greuel, G.-M.; Lossen, C.: Equianalytic and equisingular families of curves on surfaces. Manuscr. math. 91, 323-342 (1996).

[GLS] Greuel, G.-M.; Lossen, C.; Shustin, E.: Geometry of families of nodal curves on the blown-up projective plane. Trans. Amer. Math. Soc. 350, 251-274 (1998).

[GrM] Gradolato M.A.; Mezzetti E.: Families of curves with ordinary singular points on regular surfaces. Ann. mat. pura et appl. 150, 281-298 (1988).

[Hi1] Hirano, A.: Constructions of plane curves with cusps. Saitama Math. J. 10, 21-24 (1992).

[HiA] Hirschowitz, A.: Une conjecture pour la cohomologie des diviseurs sur les surfaces rationelles génériques. J. reine angew. Math. 397, 208-213 (1989).

[HiF] Hirzebruch, F.: Singularities of algebraic surfaces and characteristic numbers. Contemp. Math. 58, 141-155 (1986).

[Ivi] Ivinskis, K.: Normale Flächen und die Miyaoka-Kobayashi-Ungleichung, Diplomarbeit, Univ. Bonn (1985).

[JeL] Jensen, C.V.; Lenzing, H.: Model theoretic algebra. Gordon and Breach Sci. Publ. (1989).

[Sev] Severi, F.: Vorlesungen über algebraische Geometrie. Teubner (1921) resp. Johnson (1968).

[Shu] Shustin, E.: Real plane algebraic curves with prescribed singularities. Topology 32 no. 4, 845-856 (1993).

[Tei] Teissier, B.: Résolution simultanée I, II. In: Seminaire Demazure-Pinkham-Teissier 1976/1977, SLN 777, Springer-Verlag (1980).

[Tou] Tougeron, J.C.: Ideaux de fonctions différentiables. Ann. Inst. Fourier (Grenoble) 18 no. $1,177-240$ (1968). 
[Var] Varchenko, A.N.: Asymptotics of integrals and Hodge structures. Modern Problems of Math. 22 (Itogi nauki i tekhniki VINITI), 130-166 (Russian) (1983).

[Vir] Viro, O.Ya.: Gluing of algebraic hypersurfaces, smoothing of singularities and construction of curves. Proc. Leningrad Int. Topological Conf., 149-197 (Russian) (1983).

[Wah] Wahl, J.: Equisingular deformations of plane algebroid curves. Trans. Amer. Math. Soc. 193, 143-170 (1974).

[Wl1] Wall C. T. C. Geometry of quartic curves. Math. Proc. Camb. Phil. Soc. 117 415-423 (1995).

[W12] Wall C. T. C. Highly singular quintic curves. Math. Proc. Camb. Phil. Soc. 119 257-277 (1996).

[Zar] Zariski, O.: Studies in equisingularity I-III. Amer. Journ. Math. 87, 507-536 and 9721006 (1965), respectively Amer. Journ. Math. 90, 961-1023, (1968).

Universität Kaiserslautern, Fachbereich Mathematik, Erwin-Schrödinger-Strasse, D 67663 KAISERSLAUTERN

E-mail address: greuel@mathematik.uni-kl.de

Universität Kaiserslautern, Fachbereich Mathematik, Erwin-Schrödinger-Strasse, D 67663 KaISERSLAUtern

E-mail address: lossen@mathematik.uni-kl.de

Tel Aviv University, School of Mathematical Sciences, Ramat Aviv, ISR - Tel Aviv 69978

E-mail address: shustin@math.tau.ac.il 